\title{
Stereoselective synthesis of 3-deoxy-piperidine iminosugars from L-lysine
}

\author{
Noriaki Moriyama, Yoshihiro Matsumura, Masami Kuriyama, and Osamu Onomura* \\ Graduate School of Biomedical Sciences, Nagasaki University, 1-14 Bunkyo-machi, Nagasaki

$$
\text { 852-8521, Japan }
$$

\begin{abstract}
A new method using electrochemical oxidation and/or $\mathrm{O}_{5} \mathrm{O}_{4}$ oxidation has been exploited for the stereoselective synthesis of 2,3,6-trihydroxylated $5 S$-piperidine derivatives. The electrochemical method was successively used for the conversion of $N$-protected piperidines to $N$-protected 1-methoxypiperidines and for the conversion of 2,3-didehydro-1-methoxypiperidine derivatives to 2,3-trans-1,2,3-triacetoxypiperidine derivatives. These triacetates were easily transformed into $2 S, 3 S$,6-triacetoxy-5S-methylpiperidine and $2 R, 3 R$,6-triacetoxy-5S-methylpiperidine. In addition, 2,3-cis-dihydroxylation of 2,3-didehydro-1-methoxypiperidine derivatives with $\mathrm{OsO}_{4}$ afforded $2 R, 3 S$,6-triacetoxy-5S-methylpiperidine and $2 S, 3 R$,6-triacetoxy-5S-methylpiperidine.
\end{abstract}

\section{Introduction}

Polyhydroxylated $5 S$-methylpiperidines $\mathbf{1}$, a class of piperidine iminosugars, have attracted great interest due to their biological properties. ${ }^{1,2}$ Some of them are potential inhibitors of glycosidases and glycoprotein-processing enzymes. Now they are widely investigated as candidates for drugs to treat a variety of carbohydrate-mediated diseases such as diabetes, viral infections including HIV, and cancer metastasis. The inhibitory activities depend on the configuration and the number of hydroxyl groups. Among 1, 2,3,6-trihydroxy-5S-methylpiperidines 2 are noteworthy since recently it has been reported that $2 R, 3 S$,6-trihydroxy-5S-methylpiperidine (2a), one of the possible stereoisomers 2a-d (Fig. 2), has high inhibitory activities toward glycosidases. However, there has not been any convenient synthetic method for 2a-d. ${ }^{3,4}$ We have exploited a facile method for the stereoselective synthesis of $\mathbf{2 a - d}$, and preliminarily reported the synthesis of $\mathbf{2} \mathbf{b}, \mathbf{c}$ using electrochemical 2,3-trans-diacetoxylation. ${ }^{5}$ This paper describes the synthesis for $\mathbf{2 b , c}$ as well as those for 2a,d using 2,3-cis-dihydroxylation with $\mathrm{OsO}_{4}$.

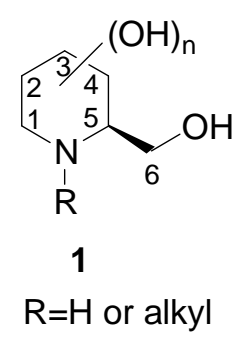

Figure 1. 

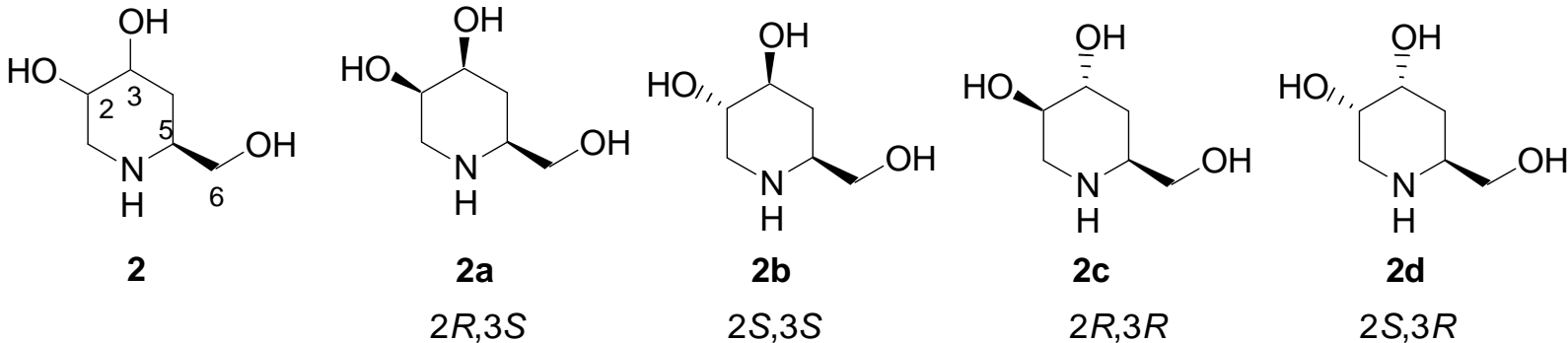

Figure 2. Stereoisomers 2a-d of 2,3,6-trihydroxy-5S-methylpiperidines 2.

\section{Result and discussion}

\subsection{Electrochemical 2,3-trans-diacetoxylation}

Our strategy to this end is based on preparation of triacetate $\mathbf{6}$, a precursor of $\mathbf{2}$, from $5 S$-acetoxymethylpiperidine derivative 3 by electrochemical oxidation; electrochemical 1-methoxylation of $\mathbf{3}$ and electrochemical triacetoxylation of $5 S$-acetoxymethyl-2,3-didehydro-1-methoxypiperidine derivative 4 (Eq. 1).

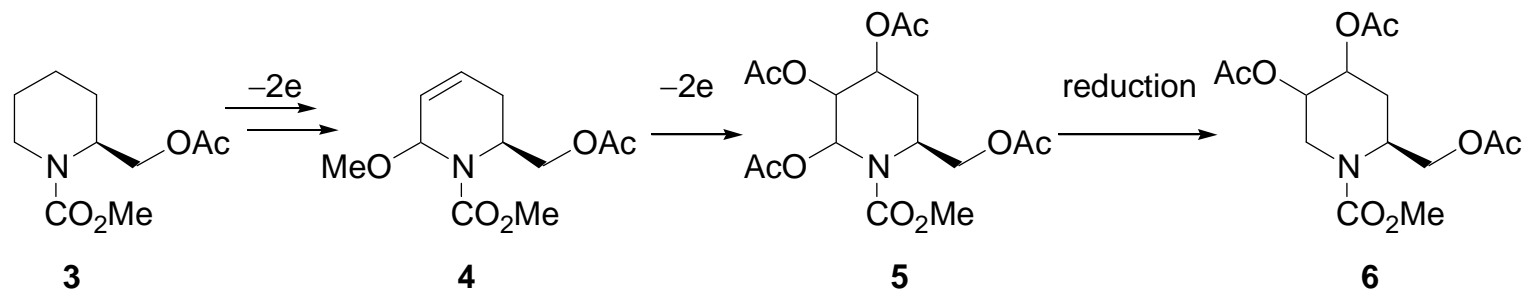

The first key electrochemical reaction in the scheme has already been used in the transformation of $\mathrm{N}$-methoxycarbonylpiperidine 7a to 2,3-didehydro-1-methoxypiperidine 10a. The transformation consisted of electrochemical oxidation of $7 \mathbf{a}$ to afford 1-methoxypiperidine $\mathbf{8 a},{ }^{6}$ elimination of $\mathrm{MeOH}$ from $\mathbf{8 a}$ to 1,2-didehydropiperidine $\mathbf{9 a}{ }^{7}$ which then underwent bromine oxidation ${ }^{8}$ followed by base-induced dehydrobromination to form 2,3-didehydro-1-methoxypiperidine $10 a$ (Eq. 2). ${ }^{9}$ The other 2,3-didehydro -1-methoxypiperidines $\mathbf{1 0 b}-\mathbf{d}$ were similarly prepared from $\mathbf{7 b - d .}$

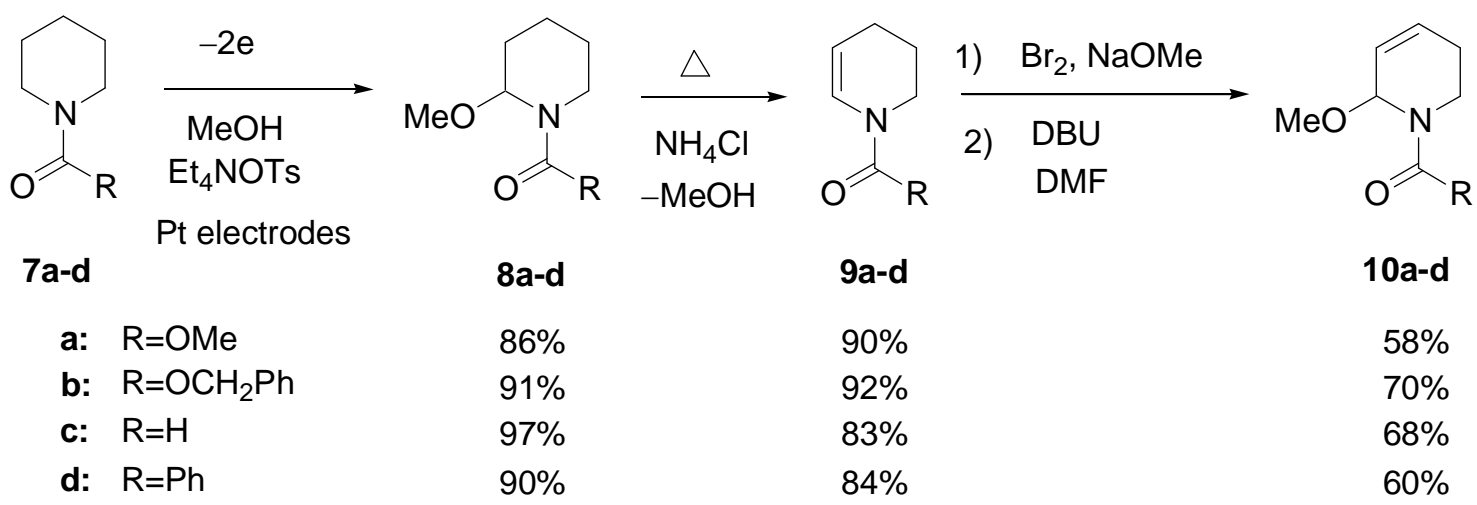


With 10a-d in hand, we examined the second key electrochemical triacetoxylation of 10a-d, which was carried out in acetic acid containing potassium acetate (Eq. 3). ${ }^{10}$ As expected, the oxidation gave triacetoxylated products 11a-d, though their stereochemistry was not determined at this stage. Then we achieved the reductive elimination of 1-acetoxyl group of 11a-d by $\mathrm{Et}_{3} \mathrm{SiH}$ to afford 2,3-diacetoxypiperidines 12a-d. The yields of 11a-d and 12a-d are shown together with the trans/cis ratio in Table 1.

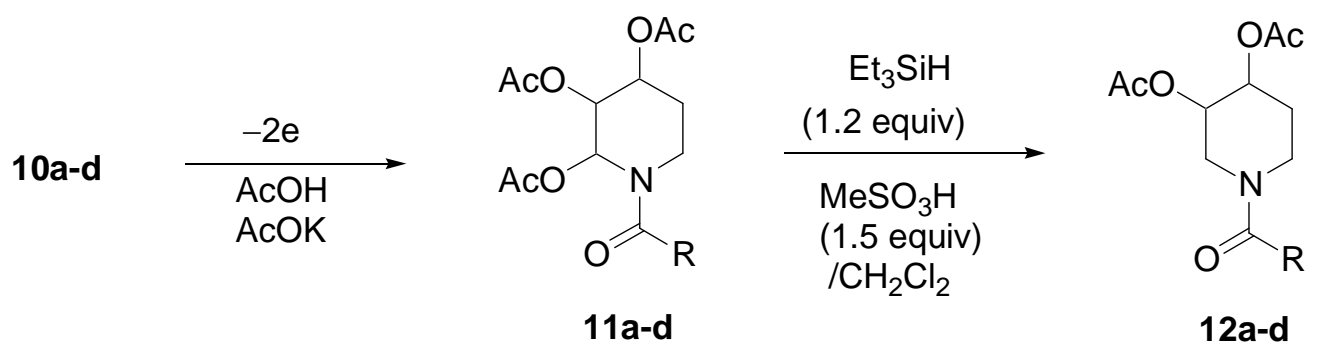

Table 1.

Electrochemical oxidation of 10a-d followed by reduction of 11a-d with $\mathrm{Et}_{3} \mathrm{SiH}$

\begin{tabular}{lllcl}
\hline Entry & 10a-d & & Yield (\%) & $\begin{array}{l}\text { trans:cis } \\
\text { (12a-d) }\end{array}$ \\
& $\mathrm{R}$ & 11a-d & 12a-d & $70: 30$ \\
\hline 1 & $\mathrm{OMe}$ & 81 & 84 & $58: 42$ \\
3 & $\mathrm{OCH}_{2} \mathrm{Ph}$ & 54 & 82 & $66: 34$ \\
4 & $\mathrm{H}$ & 78 & 65 & $54: 46$ \\
\hline
\end{tabular}

The stereochemistry (trans/cis) of 12a-d was a little bit dependent on R (70/30 54/46). ${ }^{11}$ We then, tried the preparation of $\mathbf{4}$ from easily available L-lysine derivative $13^{12}$ instead of expensive L-pipecolic acid derivative 3 through 14 and $15^{13}$ to obtain 4 in a similar way to transformation of $\mathbf{7}$ to 10. The result is shown in Scheme 1. Electrochemical oxidation of $\mathbf{4}$ under conditions similar to the oxidation of $\mathbf{1 0}$ to $\mathbf{1 1}$ afforded tetraacetoxylated piperidine $\mathbf{5}$, of which reduction with $\mathrm{Et}_{3} \mathrm{SiH}$ gave 2,3,6-triacetoxy-5S-methylpiperidine $\mathbf{6}$ as a mixture of stereoisomers. The ratio of the diastereoisomers was determined to be 91/3/3/3. 


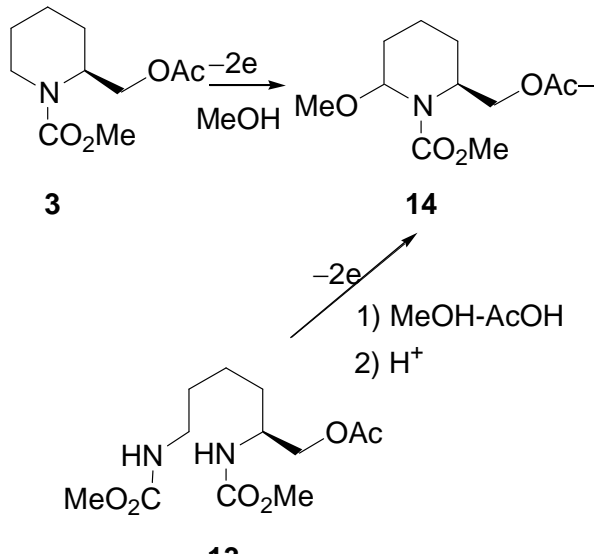

13

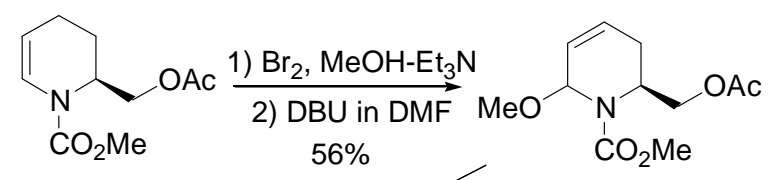

15

$80 \%$ from 3

$66 \%$ from 13

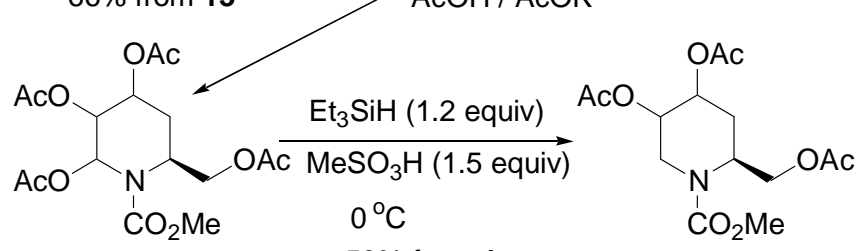

5
$53 \%$ from 4

$91: 3: 3: 3$

trans/cis $=94 / 6$

Scheme 1. Preparation of $\mathbf{6}_{2 s, 3 s, 5 s}$ starting from 3 or 13.

Fortunately, the main product $\mathbf{6}_{2 S, 3 s, 5 s}$ crystallized, and the absolute stereochemistry was determined to be $(2 S, 3 S, 5 S)$ by its X-ray analysis (Fig. 3$).^{14}$

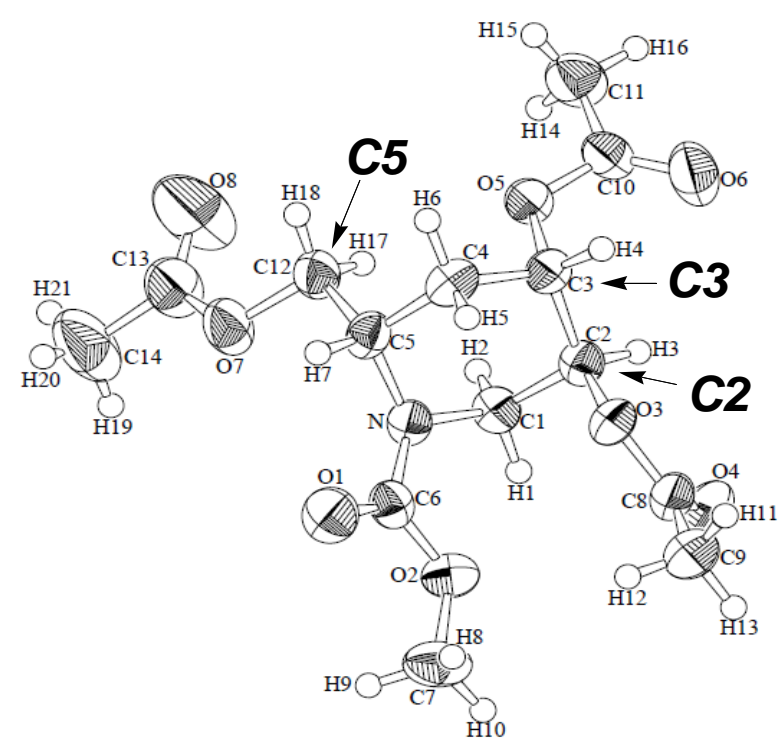

Figure 3. Ortep drawing of $\mathbf{6}_{2 S, 3 s,} 5 s$.

On the other hand, electrochemical oxidation of bicyclic carbamate 19, which was prepared from L-pipecolic acid derivative $\mathbf{1 6}$ or from L-lysine derivative $\mathbf{2 2}$ through $\mathbf{1 7}$ and 18, ${ }^{15}$ followed by reduction of the oxidation product 20 (70\% yield) with $\mathrm{Et}_{3} \mathrm{SiH}$ gave a single stereoisomer 21 (Scheme 2), of which absolute stereochemistry was also determined by its X-ray analysis (Fig. 4). ${ }^{14}$ 


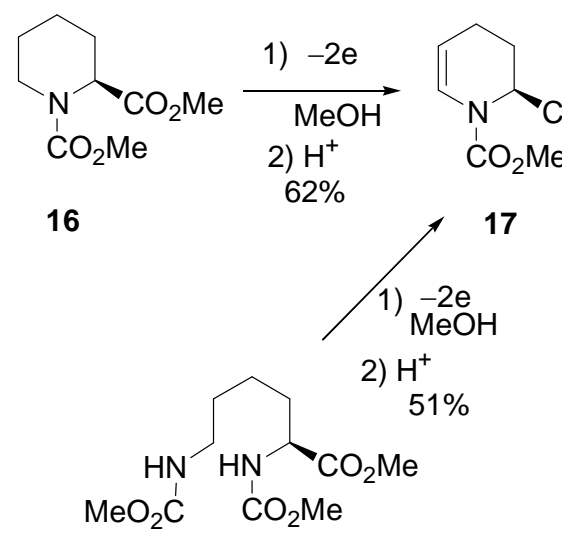

22
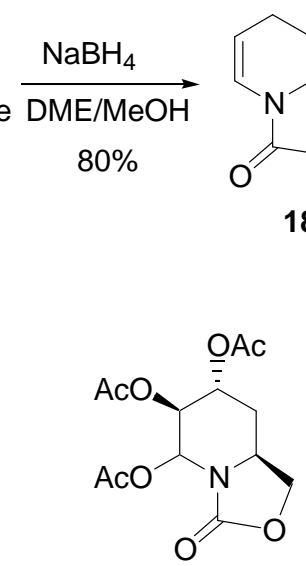

20

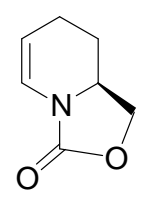

18
1) $\mathrm{Br}_{2}$, NaOMe

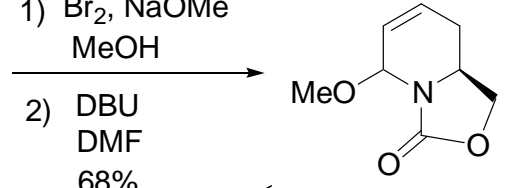

19

Scheme 2. Preparation of $\mathbf{2 1}_{2 R, 3 R, 5 S}$ starting from 16 or 22.

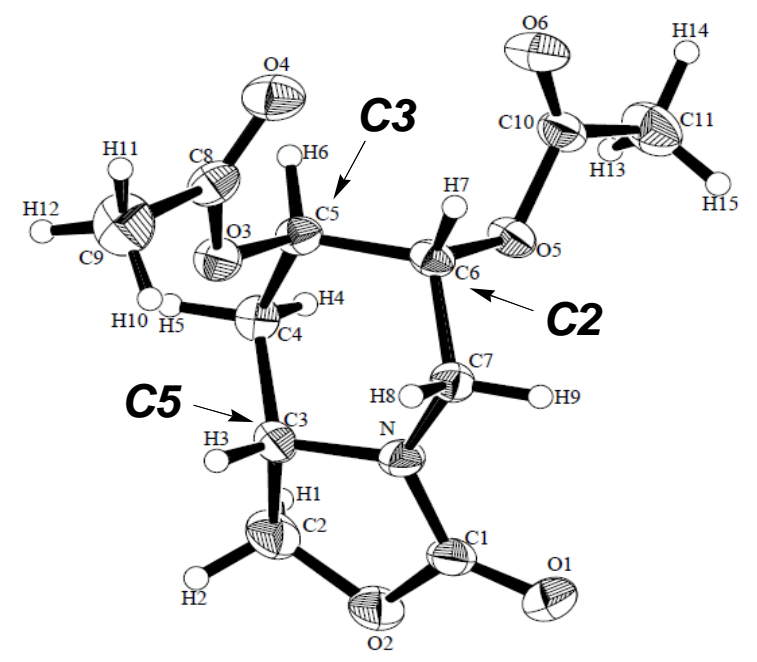

Figure 4. Ortep drawing of $\mathbf{2 1}_{2 R, 3 R, 5 s}$.

The reaction mechanism for electrochemical triacetoxylation is tentatively proposed as follows (Scheme 3). Since it was found that 10a was immediately converted to 3-acetoxy-1,2-didehydropiperidine $\mathbf{2 3}^{9 \mathrm{i}}$ under the reaction conditions, oxidation of $\mathbf{2 3}$ may be responsible for the formation of 11a by EC mechanism through dication $\mathbf{A}$ or by ECEC mechanism through cation radical $\mathbf{B}$, radical $\mathbf{C}$, and cation $\mathbf{D}{ }^{10}$ Similarly, electrochemical triacetoxylation of $\mathbf{4}$ and $\mathbf{1 9}$ may proceed via 3-acetoxypiperidine derivatives 24 and 25, respectively (Fig. 5). Since cis-isomer $\mathbf{2 4}$ was thermodynamically more stable than its trans-isomer 24', 24 should be stereospecifically formed. On the other hand, treatment of 19 with acetic acid could generate a cationic species $\mathbf{E}$, in which the endo side might be more crowded than the exo side, to afford exclusively a trans-isomer $\mathbf{2 5}$ without a cis-isomer 25, ${ }^{15 b}$ 


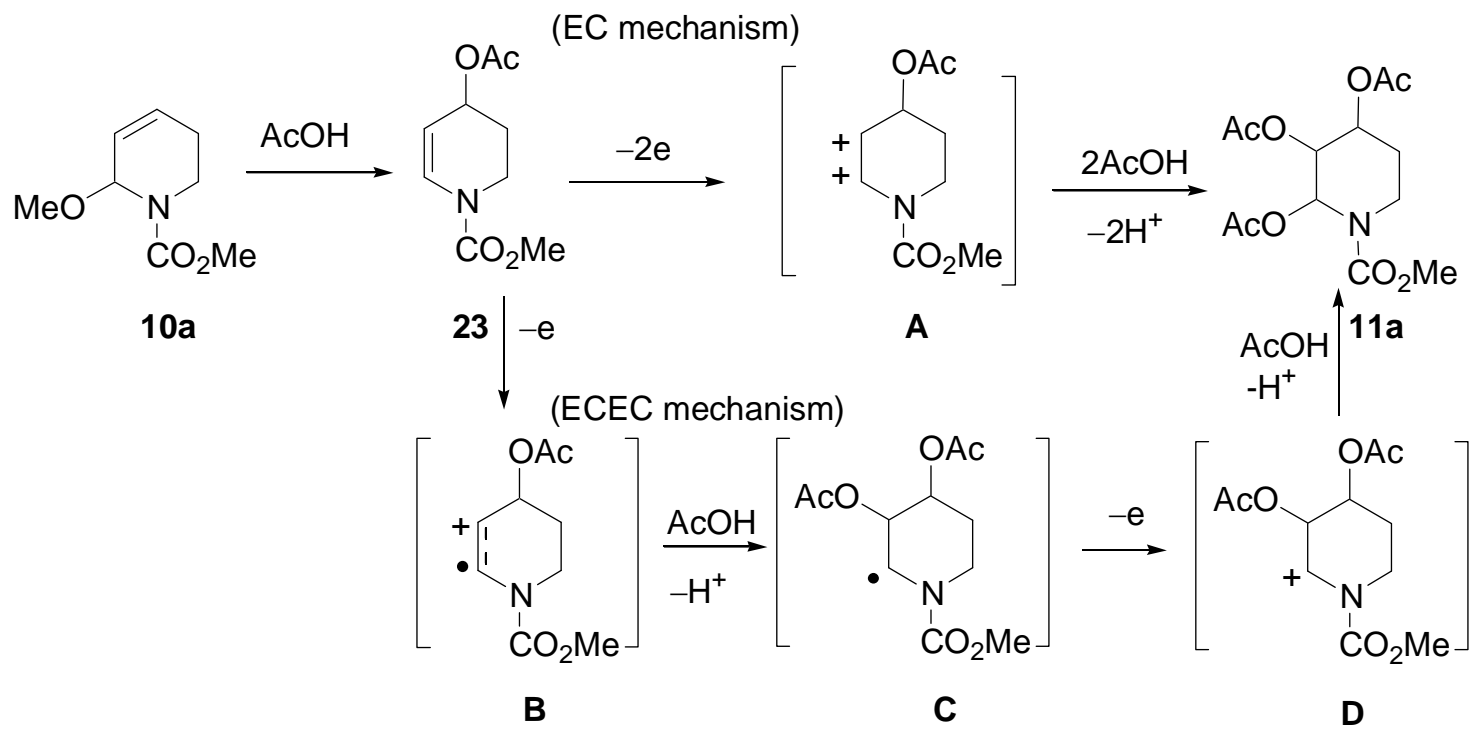

Scheme 3. Plausible mechanism for electrochemical triacetoxylation of 10a.<smiles>CC(=O)OCC1CC(OC(C)=O)C=CN1C(C)=O</smiles>

24<smiles>CC(=O)OC1C=CN2C(=O)OC[C@H]2C1</smiles>

25<smiles>CC(=O)OCC1CC(OC(C)=O)C=CN1C(C)=O</smiles>

24'<smiles>CC(=O)O[C@H]1C=CN2C(=O)OC[C@H]2C1</smiles>

25'

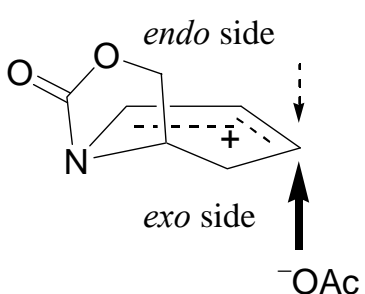

$\mathbf{E}$

Figure 5. Plausible intermediary species for electrochemical oxidation of $\mathbf{4}$ and $\mathbf{1 9}$ in $\mathrm{AcOH}$.

The oxidation potentials of some 1,2-didehydro- and 2,3-didehydro-piperidine derivatives shown in Table 2 support this proposed mechanism.

Table 2.

Oxidation potential of didehydropiperidine derivatives

\begin{tabular}{|c|c|c|c|c|c|c|}
\hline Entry & Compound & & $\begin{array}{l}\text { Oxidation } \\
\text { Potential } \\
(\mathrm{V})^{\mathrm{a}}\end{array}$ & Entry & Compound & $\begin{array}{l}\text { Oxidation } \\
\text { Potential } \\
(\mathrm{V})^{\mathrm{a}}\end{array}$ \\
\hline 1 & $\begin{array}{l}\mathrm{N}^{\prime} \\
\mathrm{CO}_{2} \mathrm{Me}\end{array}$ & $9 a$ & 1.44 & 5 & & 1.72 \\
\hline
\end{tabular}


2<smiles>COC(=O)N1CC=CCC1</smiles>

26

1.96<smiles>COC1C=CCCN1C(C)=O</smiles>

10a 1.66

3

4<smiles>CC(=O)OC1C=CN(C(C)=O)CC1</smiles><smiles>CC(=O)OCC1CC(OC(C)=O)C=CN1C(C)=O</smiles><smiles>COC1C=CC[C@@H]2COC(=O)N12</smiles>

$19 \quad 1.73$

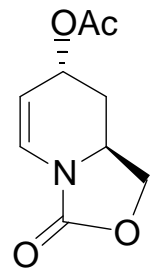

${ }^{\mathrm{a}} \mathrm{V}$ vs $\mathrm{Ag} / \mathrm{AgNO}_{3}, 0.1 \mathrm{M} \mathrm{Et}_{4} \mathrm{NClO}_{4} / \mathrm{MeCN}, 100 \mathrm{mV} / \mathrm{s}$.

A predominant formation of $\mathbf{5}_{2 S, 3 S, 5 S}$ and $\mathbf{2 0}_{2 R, 3 R, 5 S}$ may be explained by an ECEC mechanism shown in Scheme 4. As for 3-acetoxy-1,2-didehydropiperidine intermediate 24, it is possible that the plausible intermediary species could be electrochemically generated cation radical $\mathbf{F}{ }^{10 \mathrm{~b}, 16}$ Therefore, the observed high diastereoselectivity in electrochemical oxidation of $\mathbf{2 4}_{35,5 s}$ can be explained as follows: acetate ion attack on the cationic intermediate $\mathbf{F}$ is easier from the axial direction than the equatorial direction to produce $\mathbf{5}_{2 S, 3 S, 5 s}$ through the radical intermediate $\mathbf{G}$. The stereoselectivity is explainable in terms of participating effect of 3-acetoxyl group or thermodynamic control of the product. On the other hand, in the case of electrochemical oxidation of $\mathbf{2 5}_{\mathbf{3 R}, \mathbf{5}}$, acetate ion attack to cation radical $\mathbf{H}$ is easier from the equatorial direction than the axial direction to produce $\mathbf{2 0}_{2 R, 3 R, 5 s}$ through the radical intermediate $\mathbf{I}$.

The less stereoselective triacetoxylation of 10a-d may be due to a conformational flexibility of piperidine ring, which has no substituent at 5-position.
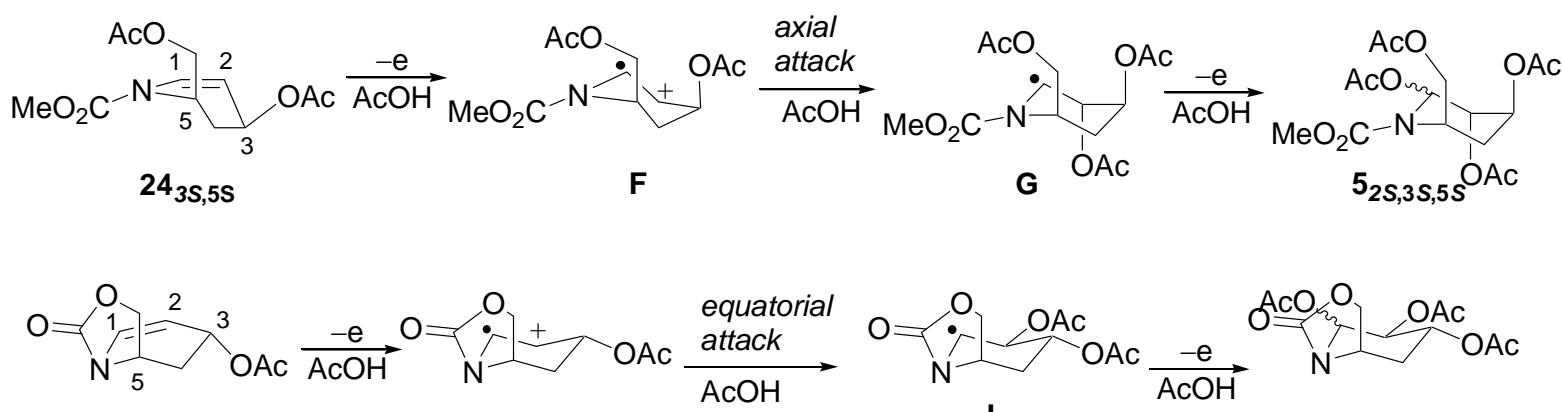

$25_{3 R, 5 S}$

H

$20_{2 R, 3 R, 5 S}$ 
Scheme 4. Plausible mechanism for electrochemical 2,3-trans-acetoxylation of 24 and 25.

\section{2 cis-Selective 2,3-dihydroxylation with $\mathrm{OsO}_{4}$}

To prepare 2,3-cis-dihydroxylated compounds $\mathbf{2 a}$ and $\mathbf{2 d}$, oxidation of $\mathbf{4}$ or $\mathbf{1 9}$ with $\mathrm{OsO}_{4}$ seems to be convenient (Scheme 5). ${ }^{2 e}$<smiles>COC(=O)COC(=O)N1C(OC)C=CC[C@@H]1COC(=O)OC</smiles>

4<smiles>CO[C@H]1C=CC[C@@H]2COC(=O)N12</smiles>

19<smiles>CC(=O)OC[C@H]1C[C@H](OC(C)=O)[C@@H](OC(C)=O)CN1C(C)=O</smiles>

$27_{2 S, 3 R, 5 S}$

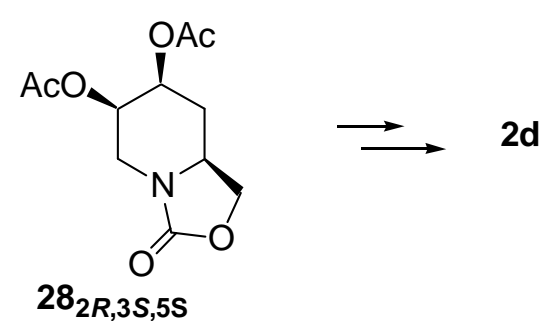

Scheme 5. Strategy for preparation of $\mathbf{2 a}$ and $\mathbf{2 d}$.

First, we investigated the $\mathrm{OsO}_{4}$ oxidation of 10a. Compound 10a was oxidized with catalytic $\mathrm{OsO}_{4}$ and 1.5 equiv of NMO followed by acetylation with acetic anhydride and pyridine to produce 2,3,4-triacetoxypiperidine 11a in 71\% yield. Compound 11a was easily reduced with $\mathrm{Et}_{3} \mathrm{SiH}$ to give cis-2,3-diacetoxypiperidine 12a (Eq. 4).

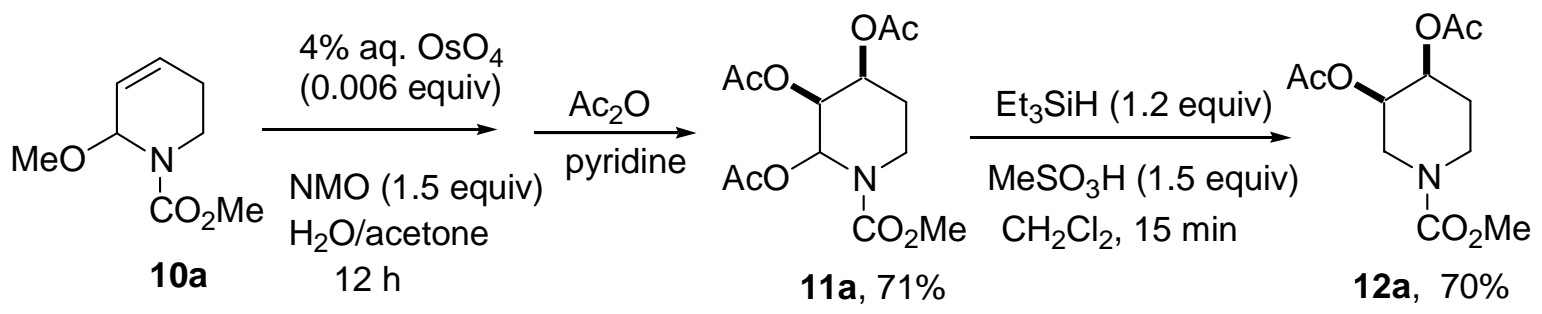

Encouraged by this result, we continuously tried to apply the same conditions to $5 S$-acetoxymethylpiperidine derivatives 4 (Scheme 6). As expected, the $\mathrm{OsO}_{4}$ oxidation and subsequent acetylation proceeded smoothly, but the reaction product was a mixture of 2,3-diacetoxy-5S-acetoxymethyl-1-methoxy- $N$-methoxycarbonylpiperidine $\quad$ 29a and 1,2,3-triacetoxy-5S-acetoxymethyl- $N$-methoxycarbonylpiperidine 29b. Without purification of the mixture, reduction with $\mathrm{Et}_{3} \mathrm{SiH}$ was carried out to provide only one product, 2,3-diacetoxy-5S-acetoxymethyl- $N$-methoxycarbonylpiperidine 27 . Since 27 did not 
crystallize, we tried to prepare its tosylated derivatives to determine absolute stereochemistry of the two hydroxyl groups at the 2,3-position by X-ray analysis.

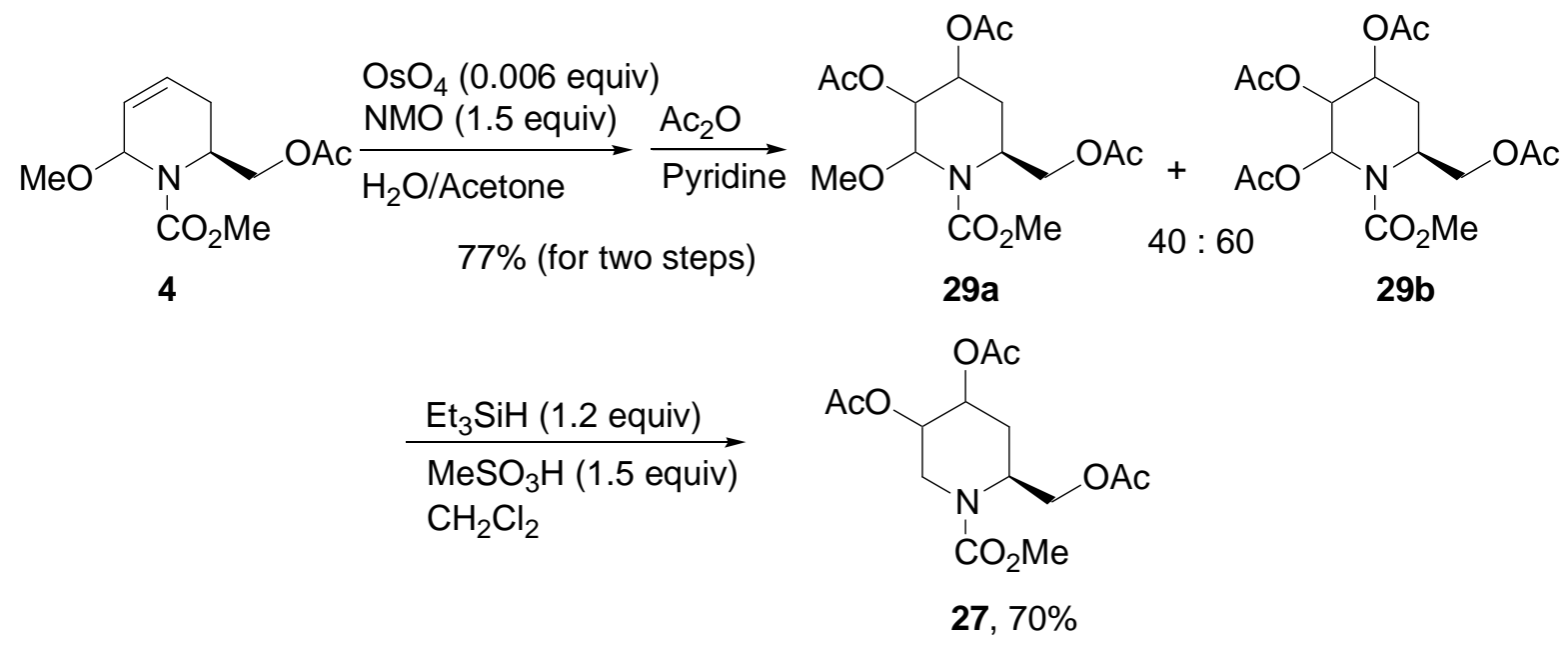

Scheme 6. Preparation of 27.

The $\mathrm{OsO}_{4}$ oxidation of 4 and successive reduction with $\mathrm{Et}_{3} \mathrm{SiH}$ gave 2,3-dihydroxylated derivative $\mathbf{3 0}$ as a single diastereomer (Scheme 7). Then, compound $\mathbf{3 0}$ was treated with tosyl chloride to afford crystal 2,3-ditosyloxylated derivative 31. The X-ray analysis of compound 31 determined its absolute stereoconfiguration, $(2 S, 3 R, 5 S) .{ }^{14}$

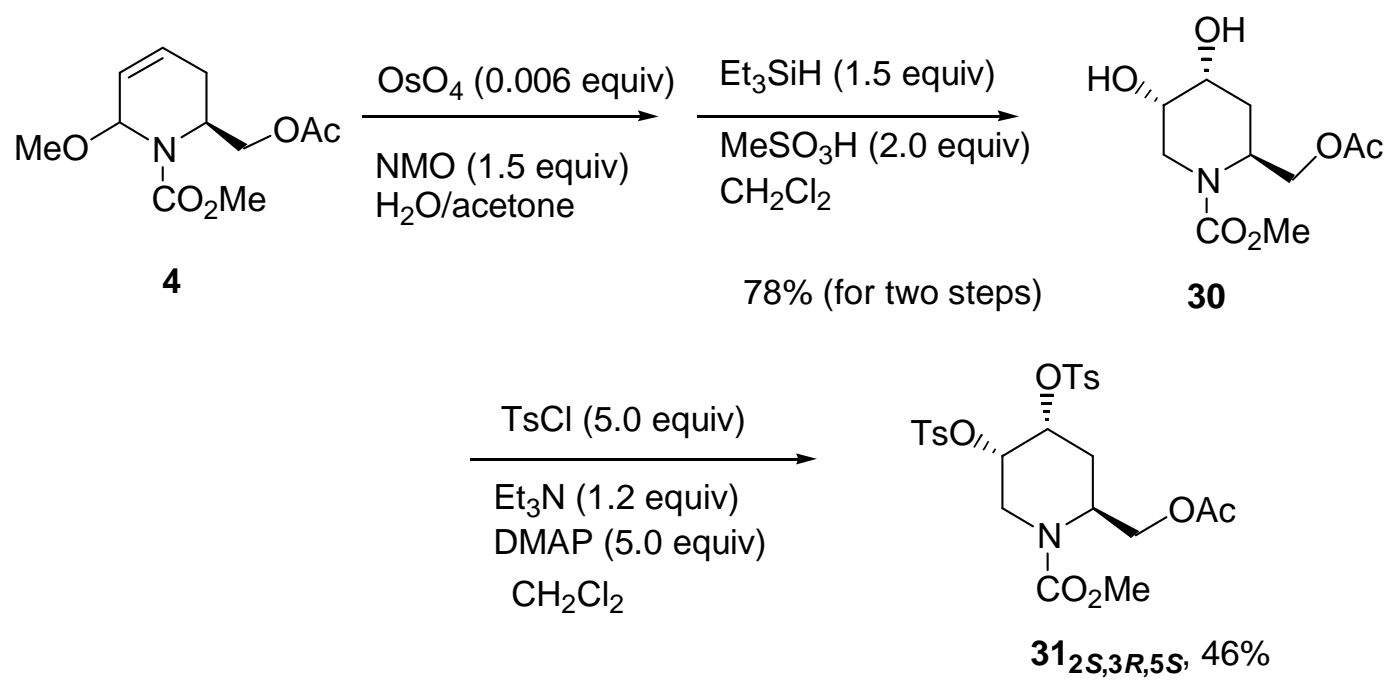

Scheme 7. Preparation of $\mathbf{3 1} 2 S, 3 R, 5 s$. 


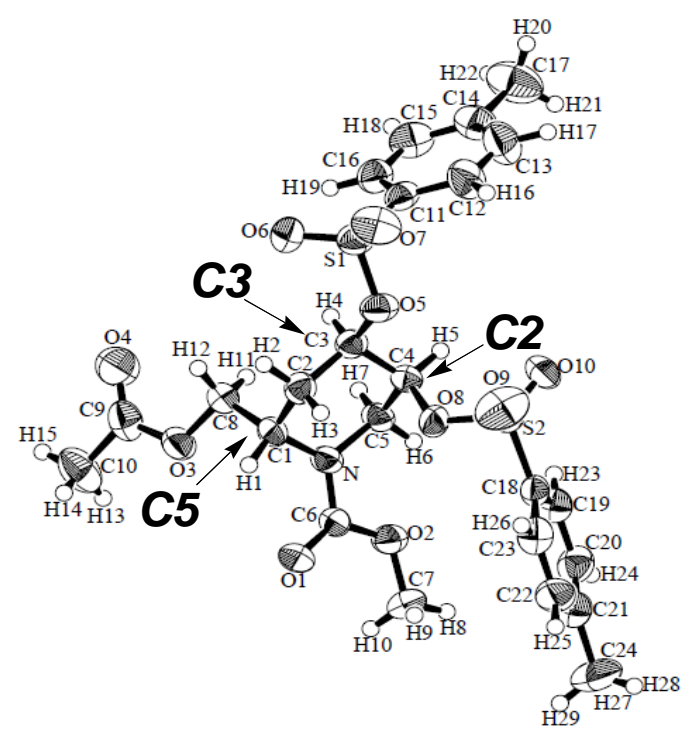

Figure 6. Ortep drawing of $\mathbf{3 1} 1_{2 s, 3 R, 5 s}$.

Next, the $\mathrm{OsO}_{4}$ oxidation of bicyclic carbamate 19 and successive acetylation with $\mathrm{Ac}_{2} \mathrm{O}$-pyridine was examined to give 1-methoxy-2,3-diacetoxylated compound 32. In this case, 1-methoxy group remained unchanged in this reaction condition. Finally, compound 32 was reduced by $\mathrm{Et}_{3} \mathrm{SiH}$ to afford 2,3-diacetoxylated bicyclic carbamate $\mathbf{2 8}$ as a single diastereomer (Eq. 5). The absolute stereoconfiguration of $\mathbf{2 8}$ was determined by X-ray analysis to be $2 R, 3 S, 5 S$ (Fig. 7). ${ }^{14}$<smiles>COC1C=CCC2COC(=O)N21</smiles>
19
1) $\mathrm{OsO}_{4}$ (0.006 equiv) $\mathrm{NMO}$ (1.5 equiv) $\mathrm{H}_{2} \mathrm{O} /$ Acetone 2) $\mathrm{Ac}_{2} \mathrm{O}$-Pyridine<smiles>COC1[C@H](OC(C)=O)[C@@H](OC(C)=O)C[C@H]2CO[C@H](O2)N1C(C)=O</smiles>

32, $85 \%$

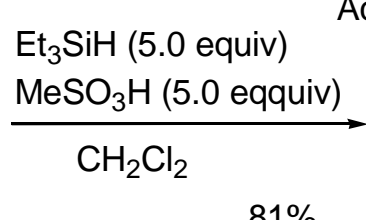<smiles>CC(=O)OC1CN2C(=O)OC[C@H]2C[C@H]1OC(C)=O</smiles>

$28_{2 R, 3 S, 5 S}$ 


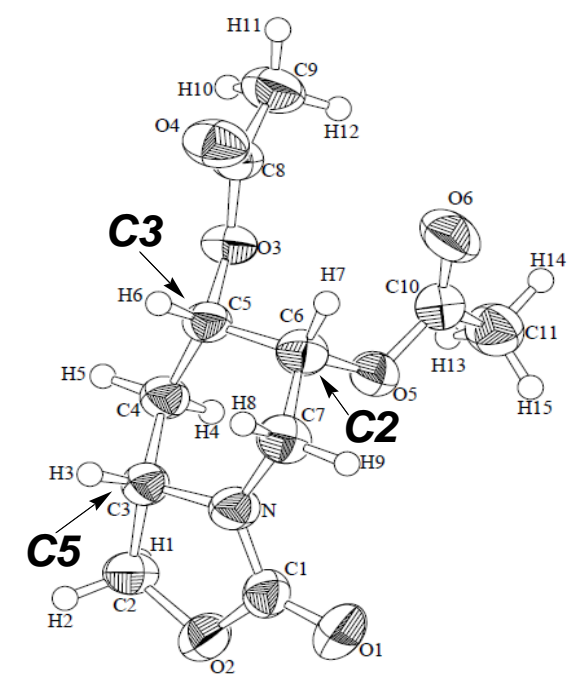

Figure 7. Ortep drawing of $\mathbf{2 8}_{2 R, 3 S, 5 s}$.

The observed high diastereoselectivity by the $\mathrm{OsO}_{4}$ oxidation in this case can be explained by anomeric effect of 1-methoxyl group. That is, since the methoxyl group is mainly located at the axial position, it is difficult for $\mathrm{OsO}_{4}$ to get close to 19 from down side (approach $\mathrm{B}$ ), while $\mathrm{OsO}_{4}$ can easily get close to 19 from the upper side (approach A) (Scheme 8). Accordingly, the $\mathrm{OsO}_{4}$ oxidation of 19 and successive reduction exclusively afford dihydroxylated compound $\mathbf{J}$ as a precursor for $\mathbf{2 8}_{2 \mathbf{R}, 3 S, 5 S}$.

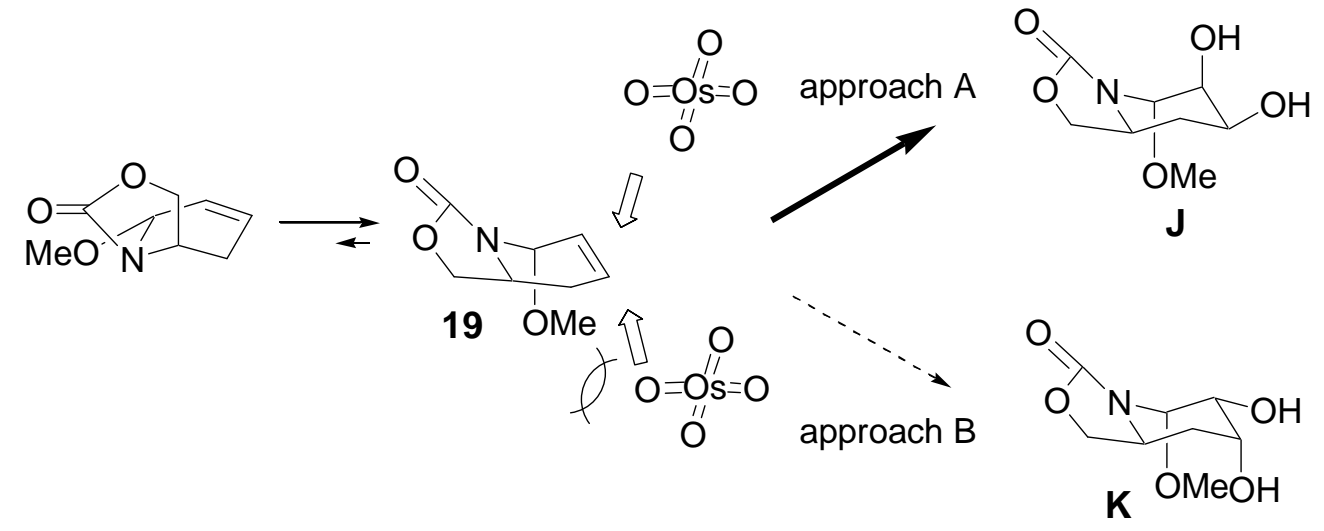

Scheme 8. Effect of methoxyl group at the 1-position of 19.

Next, bicyclic carbamate 33, which has no 1-methoxyl group, was examined. $\mathrm{OsO}_{4}$ oxidation of 33 followed by acetylation afforded a mixture of $2 R, 3 S$-isomer $\mathbf{2 8}_{2 R, 3 S, 5 S}$ and

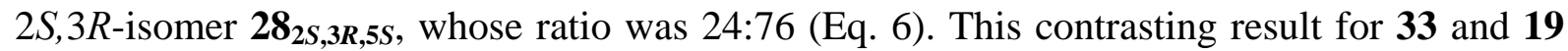
supports our proposed stereochemical course shown in Scheme 8. The result can represent the importance of the steric effect of 1-methoxyl group on the observed high diastereoselectivity. 


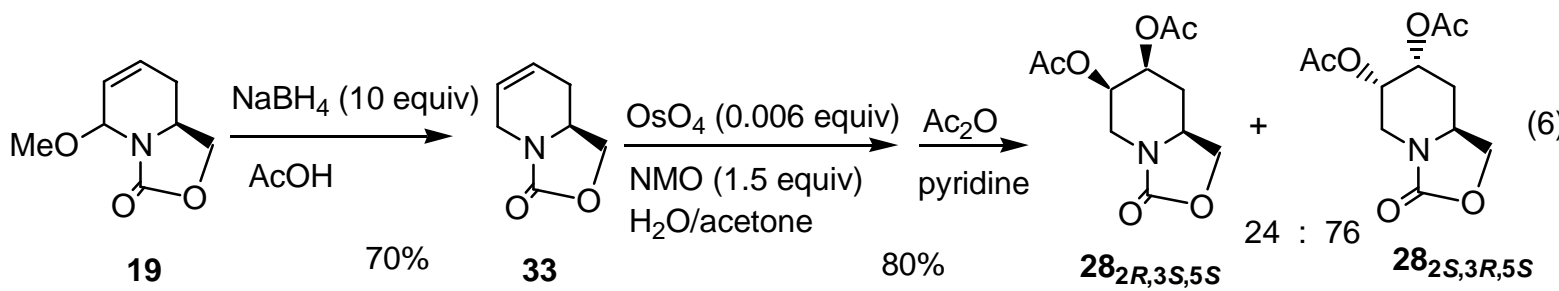

In summary, the stereoselective formal syntheses of 2,3,6-trihydroxylated $5 S$-methylpiperidines 2a-d from L-lysine and L-pipecolic acid has been accomplished by using tandem electrochemical oxidation or $\mathrm{OsO}_{4}$ oxidation.

\section{Experimental Section}

\subsection{General}

Electrochemical reactions were carried out using DC Power Supply (GP 050-2) of Takasago Seisakusho, Inc. ${ }^{1} \mathrm{H}$ NMR spectra were measured on a Varian Gemini 300 spectrometer with TMS as an internal standard. IR spectra were obtained on a Shimadzu FTIR-8100A. Mass spectra were obtained on a JEOL JMS-DX 303 instrument. HPLC analyses were achieved by using a LC-10AT VP and a SPD-10A VP of Shimadzu Seisakusho, Inc. Specific rotations were measured with JASCO DIP-1000. Melting points are uncorrected. Elemental analyses were carried out at the Center for Instrumental Analysis, Nagasaki University.

All reagents and solvents were used as supplied without further purification.

\subsection{Measurement of oxidation potentials}

BAS CV-50W was used as a voltametric analyzer. A solution of substrate $(0.1 \mathrm{mmol})$ in MeCN (10 mL) containing $0.1 \mathrm{M} \mathrm{Et}_{4} \mathrm{NBF}_{4}$ was measured. Reference electrode was $\mathrm{Ag} / \mathrm{AgNO}_{3}$ in saturated aqueous $\mathrm{KCl}$, a working electrode was a glassy carbon, and a counter electrode was a platinum wire. Scan rate was $100 \mathrm{mV} / \mathrm{s}$.

\subsection{Preparation of 2,3-didehydro-1-methoxy- $N$-acylpiperidines $10 \mathrm{a}-\mathrm{d}$}

Transformations of 1-acylpiperidines 7a-d to 2,3-didehydro-1-methoxy- $N$-acylpiperidines $\mathbf{1 0 a}-\mathbf{d}$ were carried out according to our reported method. ${ }^{9}$ Compounds $\mathbf{8 a},{ }^{6 \mathrm{a}} \mathbf{8 b},{ }^{6 \mathrm{c}} \mathbf{8 c},{ }^{6 \mathrm{~b}} \mathbf{8 d},{ }^{9 \mathrm{c}}$ $\mathbf{9 a},{ }^{7 \mathrm{~b}} \mathbf{9 b},{ }^{7 \mathrm{~d}} \mathbf{9 c},{ }^{7 \mathrm{a}} \mathbf{9 d},{ }^{7 \mathrm{c}} \mathbf{1 0 a},{ }^{9 \mathrm{~b}}$ and $\mathbf{1 0 d ^ { 9 \mathrm { d } }}$ are known. 
The characterization data for unknown compounds $10 \mathrm{~b}$ and 10c are described below.

$N$-Benzyloxycarbonyl-2,3-didehydro-1-methoxypiperidine (10b): ${ }^{1} \mathrm{H}-\mathrm{NMR} \quad\left(\mathrm{CDCl}_{3}\right) \delta$ 1.92-2.05 (m, 1H), 2.10-2.30 (m, 1H), 3.05-3.25 (m, 1H), 3.29 and 3.39 (2s, 3H), 4.02-4.25 (m, 1H), 5.12-5.26 (m, 2H), 5.40-5.55 (m, 1H), 5.70-5.84 (m, 1H), 5.95-6.06 (m, 1H), 7.36 (s, 5H); IR (neat) 3038, 2936, 1713, 1655, 1428, 1200, 1082, 982, $698 \mathrm{~cm}^{-1}$; HRMS (EI) $\mathrm{m} / \mathrm{z}$ Calcd for $\mathrm{C}_{14} \mathrm{H}_{17} \mathrm{NO}_{3}\left(\mathrm{M}^{+}\right)$: 247.1208. Found: 247.1181.

2,3-Didehydro- $\boldsymbol{N}$-formyl-1-methoxypiperidine (10c): ${ }^{1} \mathrm{H}-\mathrm{NMR}\left(\mathrm{CDCl}_{3}\right) \delta$ 2.02-2.35 (m, 2H), 2.98 (td, $J=13.1$ and $6.0 \mathrm{~Hz}, 2 / 3 \mathrm{H}), 3.30$ and 3.39 (2s, $2 \mathrm{H}$ and $1 \mathrm{H}), 3.45-3.52$ (m, 2/3H), 4.35 (dd, $J=13.5$ and $6.4 \mathrm{~Hz}, 2 / 3 \mathrm{H}$ ), 4.75 and 5.63 (2d, $J=3.0$ and $3.0 \mathrm{~Hz}, 2 / 3 \mathrm{H}$ and 1/3H), 5.78-5.88 (m, 1H), 5.92-6.10 (m, 1H), 8.26 and 8.29 (2s, 1/3H and 2/3H); IR (neat) 3567, 2938, 1692, 1655, 1433, 1084, 957, $669 \mathrm{~cm}^{-1}$; HRMS (EI) $m / z$ Calcd for $\mathrm{C}_{7} \mathrm{H}_{11} \mathrm{NO}_{2}\left(\mathrm{M}^{+}\right)$: 141.0790. Found: 141.0770 .

\section{Preparation of optically active 2,3-didehydro-1-methoxy- $N$-methoxycarbonylpiperidine} (4)

Compound 4 was prepared from L-lysine derivative 13 or L-pipecolic acid derivative 3 by our reported method. ${ }^{12 \mathrm{~b}}$ Compound $\mathbf{1 4}$ was transformed into compound $\mathbf{1 5}$ without purification. The characterization data for compounds $3, \mathbf{4 , 1 3}$, and 15 are described below.

5S-Acetoxymethyl- $N$-methoxycarbonylpiperidine (3): $[\alpha]^{28}{ }_{\mathrm{D}}-45.6$ (c 1.1, $\left.\mathrm{CHCl}_{3}\right)$; ${ }^{1} \mathrm{H}-\mathrm{NMR}\left(\mathrm{CDCl}_{3}\right) \delta$ 1.34-1.55 (m, 2H), 1.58-1.74 (m, 4H), 2.04 (s, 3H), 2.88 (t, $J=12.9 \mathrm{~Hz}$, 1H), 3.69 (s, 3H), 4.00-4.10 (m, 1H), 4.15 (dd, $J=11.4$ and $6.6 \mathrm{~Hz}, 1 \mathrm{H}), 4.24$ (dd, $J=11.4$ and $8.7 \mathrm{~Hz}, 1 \mathrm{H}$ ), 4.51 (br s, 1H); IR (neat) 2944, 1748, 1655, 1449, 1262, 1049, 841, 770 $\mathrm{cm}^{-1}$; HRMS (EI) $m / z$ Calcd for $\mathrm{C}_{10} \mathrm{H}_{17} \mathrm{NO}_{4}\left(\mathrm{M}^{+}\right)$: 215.1157. Found: 215.1146.

$5 S$-Acetoxymethyl-2,3-didehydro-1-methoxy- $N$-methoxycarbonylpiperidine (4): $[\alpha]^{28}$ +71.6 (c 1.0, $\left.\mathrm{CHCl}_{3}\right) ;{ }^{1} \mathrm{H}-\mathrm{NMR}\left(\mathrm{CDCl}_{3}\right) \delta 2.06$ (s, 3H), 2.08-2.17 (m, 1H), 2.28-2.46 (m, 1H), 3.37 and 3.42 (2br s, 3H), 3.77 (s, 3H), 4.09-4.26 (m, 2H), 4.57-4.85 (m, 1H), 5.34-5.61 (m, 1H), 5.72-5.94 (m, 2H); IR (neat) 2957, 1744, 1709, 1445, 1368, 1231, 1123, 1082, 980, 770 $\mathrm{cm}^{-1}$; HRMS (EI) $m / z$ Calcd for $\mathrm{C}_{11} \mathrm{H}_{17} \mathrm{NO}_{5}\left(\mathrm{M}^{+}\right)$: 243.1107. Found: 243.1090 .

5S-Acetoxymethyl-1,2-didehydro- $\boldsymbol{N}$-methoxycarbonylpiperidine (15): $[\alpha]^{27}{ }_{\mathrm{D}}-72.2(c 1.2$, 
methanol); ${ }^{1} \mathrm{H}-\mathrm{NMR}\left(\mathrm{CDCl}_{3}\right) \delta$ 1.69-2.07 (m, 4H), 2.06 (s, 3H), 3.77 (s, 3H), 4.01 (dd, $J=$ 10.8 and 7.2 Hz, 1H), 4.06-4.22 (m, 1H), 4.45-4.70 (m, 1H), 4.82-5.02 (m, 1H), 6.71 and 6.85 (2d, $J=8.7$ and $9.0 \mathrm{~Hz}, 1 \mathrm{H}$ ); IR (neat) 2965, 1742, 1712, 1660, 1448, 1362, $1240 \mathrm{~cm}^{-1}$; Anal. Calcd for $\mathrm{C}_{10} \mathrm{H}_{15} \mathrm{NO}_{4}$ : C, 56.33; H, 7.09; N, 6.57. Found: C, 56.07; H, 7.17; N, 6.40.

2S,6-Bis(methoxycarbonylamino)hexyl acetate (13): $[\alpha]_{D}^{28}+17.1$ (c 1.0, methanol); mp 97-98 ${ }^{\circ} \mathrm{C} ;{ }^{1} \mathrm{H}-\mathrm{NMR}\left(\mathrm{CDCl}_{3}\right) \delta$ 1.35-1.60 (m, 6H), 2.07 (s, 3H), 3.10-3.26 (m, 2H), 3.66 (s, 3H), 3.67 (s, 3H), 3.82-3.93 (m, 1H), 4.04-4.12 (m, 2H), 4.64-4.84 (m, 2H); IR (KBr) 3335, 2980, 1755, 1700, 1555, 1230, $1068 \mathrm{~cm}^{-1}$; Anal. Calcd for $\mathrm{C}_{12} \mathrm{H}_{22} \mathrm{~N}_{2} \mathrm{O}_{6}$ : C, 49.65; $\mathrm{H}, 7.64 ; \mathrm{N}$, 9.65. Found: C, 49.38; H, 7.79; N, 9.90.

\section{Preparation of optically active bicyclic compound 19}

Compound 19 was prepared from L-lysine derivative $22^{12 a}$ or L-pipecolic acid derivative 16 by procedures similar to preparation of 4 .

The characterization data for compounds $\mathbf{1 6}, \mathbf{1 7}, \mathbf{1 8},{ }^{15} \mathbf{1 9},{ }^{15}$ and 22 are described below.

5S,N-Bis(methoxycarbonyl)piperidine (16): $[\alpha]^{25}{ }_{\mathrm{D}}-60.9$ (c 1.5 , methanol); ${ }^{1} \mathrm{H}-\mathrm{NMR}$ $\left(\mathrm{CDCl}_{3}\right) \delta$ 1.16-1.52 (m, 2H), 1.58-1.75 (m, 3H), 2.16-2.30 (m, 1H), 2.88-3.11 (m, 1H), 3.73 (s, 3H), 3.74 (s, 3H), 3.92-4.19 (m, 1H), 4.75-4.99 (m, 1H); IR (neat) 2950, 1750, 1710, 1450, 1265, 1210, 1170, $1095 \mathrm{~cm}^{-1}$; Anal. Calcd for $\mathrm{C}_{9} \mathrm{H}_{15} \mathrm{NO}_{4}$ : C, 53.72; H, 7.51; N, 6.96. Found: C, 53.70; H, 7.74; N, 6.67.

1,2-Didehydro-5S, $\boldsymbol{N}$-bis(methoxycarbonyl)piperidine (17): $[\alpha]_{\mathrm{D}}^{27}-46.9\left(c\right.$ 1.0, $\left.\mathrm{CHCl}_{3}\right)$; ${ }^{1} \mathrm{H}-\mathrm{NMR}\left(\mathrm{CDCl}_{3}\right) \delta$ 1.83-2.05 (m, 3H), 2.30-2.42 (m, 1H), $3.74(\mathrm{~s}, 3 \mathrm{H}), 3.75$ and 3.80 (2s, $2 \mathrm{H}$ and $1 \mathrm{H}), 4.81-4.91(\mathrm{~m}, 1 \mathrm{H}), 4.93-5.02(\mathrm{~m}, 1 \mathrm{H}), 6.81$ and $6.94(2 \mathrm{~d}, J=9.0$ and $8.7 \mathrm{~Hz}, 2 / 3 \mathrm{H}$ and 1/3H); IR (neat) 2950, 1755, 1720, 1445, $1360 \mathrm{~cm}^{-1}$; Anal. Calcd for $\mathrm{C}_{9} \mathrm{H}_{13} \mathrm{NO}_{4}$ : C, 54.26; H, 6.58; N, 7.03. Found: C, 54.17; H, 6.73; N, 6.74.

(6S)-1-Aza-2,3-didehydro-8-oxabicyclo[4.3.0]nonan-9-one (18): $[\alpha]_{D}^{28}+164.9$ (c 1.0, $\mathrm{CHCl}_{3}$ ); mp 45-46 ${ }^{\circ} \mathrm{C} ;{ }^{1} \mathrm{H}-\mathrm{NMR}\left(\mathrm{CDCl}_{3}\right) \delta$ 1.50-1.80 (m, 1H), 2.05-2.32 (m, 3H), 3.95-4.15 (m, 2H), 4.50-4.70 (m, 1H), 5.03-5.15 (m, 1H), 6.60 (d, $J=10.0 \mathrm{~Hz}, 1 \mathrm{H})$; IR (KBr) 1752, 1720, 1445, $1360 \mathrm{~cm}^{-1}$; Anal. Calcd for $\mathrm{C}_{7} \mathrm{H}_{9} \mathrm{NO}_{2}$ : C, 60.43; $\mathrm{H}, 6.51 ; \mathrm{N}, 10.07$. Found: C, 60.16; H, 6.56; N, 9.90. 
(6S)-1-Aza-3,4-didehydro-2-methoxy-8-oxabicyclo[4.3.0]nonan-9-one (19): $[\alpha]^{28}-226.6$ (c 1.0, $\mathrm{CHCl}_{3}$ ); mp 34-36 ${ }^{\circ} \mathrm{C} ;{ }^{1} \mathrm{H}-\mathrm{NMR}\left(\mathrm{CDCl}_{3}\right) \delta$ 2.09-2.35 (m, 2H), 3.45 (s, 3H), 3.92-4.04 (m, 1H), 4.09 (dd, $J=8.7$ and $3.6 \mathrm{~Hz}, 1 \mathrm{H}$ ), 4.56 (t, $J=8.4 \mathrm{~Hz}, 1 \mathrm{H}$ ), 5.14 (d, $J=1.2 \mathrm{~Hz}, 1 \mathrm{H}$ ), 5.81-5.91 (m, 1H), 5.94-6.02 (m, 1H); IR (KBr) 2982, 1767, 1414, 982, $763 \mathrm{~cm}^{-1}$; HRMS (EI) $m / z$ Calcd for $\mathrm{C}_{8} \mathrm{H}_{11} \mathrm{NO}_{3}\left(\mathrm{M}^{+}\right):$169.0739. Found: 169.0731 .

Methyl 2S,6-Bis(methoxycarbonylamino)hexanoate (22): $[\alpha]^{28}{ }_{\mathrm{D}}+16.5\left(c\right.$ 1.0, $\left.\mathrm{CHCl}_{3}\right)$; mp 50-51 ${ }^{\circ} \mathrm{C}$ (uncorrected); ${ }^{1} \mathrm{H}-\mathrm{NMR}\left(\mathrm{CDCl}_{3}\right) \delta$ 1.27-1.44 (m, 2H), 1.46-1.59 (m, 2H), 1.62-1.76 (m, 1H), 1.78-1.90 (m, 1H), 3.15-3.20 (m, 2H), 3.66 (s, 3H), 3.69 (s, 3H), 3.74 (s, 3H), 4.31-4.39 (m, 1H), 4.77 (br s, 1H), 5.31 (br s, 1H); IR (KBr) 3290, 2950, 1730, 1695, 1550, $1275 \mathrm{~cm}^{-1}$; Anal. Calcd for $\mathrm{C}_{11} \mathrm{H}_{20} \mathrm{~N}_{2} \mathrm{O}_{6}$ : C, 47.82; H, 7.30; N, 10.14. Found: C, 48.05; H, $7.40 ; \mathrm{N}, 10.27$.

Preparation of racemic 3-acetoxy-1,2-didehydro- $N$-methoxycarbonylpiperidine 23, and optically active 3-acetoxy-1,2-didehydro- $N$-acylpiperidines 24 and 25

Compounds 10a, 4, and 19 were easily transformed into 3-acetoxylated derivative 23, 24 and 25 by stirring in acetic acid for a few minutes with quantitative yield.

3-Acetoxy-1,2-didehydro- $N$-methoxycarbonylpiperidine (23) : ${ }^{1} \mathrm{H}-\mathrm{NMR} \quad\left(\mathrm{CDCl}_{3}\right) \delta$ 1.83-2.03 (m, 2H), 2.05 (s, 3H), 3.30-3.45 (m, 1H), 3.79 (s, 3H), 3.87-4.10 (m, 1H), 4.97-5.15 (m, 1H), 5.17-5.25 (m, 1H), 6.97 and 7.11 (2br d, $J=9.2 \mathrm{~Hz}, 1 \mathrm{H}$ ); IR (neat) 2957, 1717, 1648, 1447, 1364, 1235, 1007, $768 \mathrm{~cm}^{-1}$; HRMS $\left(\mathrm{M}^{+}\right) \mathrm{m} / z$ Calcd for $\mathrm{C}_{9} \mathrm{H}_{13} \mathrm{NO}_{4}\left(\mathrm{M}^{+}\right)$: 199.0845 . Found: 199.0822.

3S-Acetoxy-5S-acetoxymethyl-1,2-didehydro- $N$-methoxycarbonylpiperidine (24): ${ }^{1} \mathrm{H}-\mathrm{NMR}\left(\mathrm{CDCl}_{3}\right) \delta$ 1.93-2.23 (m, 1H), 2.02 (s, 3H), 2.05 (s, 3H), 2.18-2.30 (m, 1H), 3.80 (s, $3 \mathrm{H}), 4.15-4.31(\mathrm{~m}, 2 \mathrm{H}), 4.53-4.78(\mathrm{~m}, 1 \mathrm{H}), 5.02-5.24(\mathrm{~m}, 2 \mathrm{H}), 6.95$ and 7.09 (2d, $J=7.0$ and $6.4 \mathrm{~Hz}, 1 \mathrm{H}$ ); IR (neat) 2959, 1752, 1648, 1447, 1334, 1073, $768 \mathrm{~cm}^{-1}$; HRMS (EI) $m / z$ Calcd for $\mathrm{C}_{12} \mathrm{H}_{17} \mathrm{NO}_{6}\left(\mathrm{M}^{+}\right)$: 271.1056. Found: 271.1066.

(4R,6S)-1-Aza-4-acetoxy-2,3-didehydro-8-oxabicyclo[4.3.0]nonan-9-one (25): $\mathrm{mp}$ 77-79 ${ }^{\circ} \mathrm{C}$; ${ }^{1} \mathrm{H}-\mathrm{NMR}\left(\mathrm{CDCl}_{3}\right) \delta 1.72(\mathrm{td}, J=12.8$ and $3.8 \mathrm{~Hz}, 1 \mathrm{H}), 2.06(\mathrm{~s}, 3 \mathrm{H}), 2.24$ (d, $J=12.8 \mathrm{~Hz}$, 
1H), 4.01 (t, $J=9.0 \mathrm{~Hz}, 1 \mathrm{H}), 4.07-4.21(\mathrm{~m}, 1 \mathrm{H}), 4.67$ (t, $J=8.1 \mathrm{~Hz}, 1 \mathrm{H}), 5.25-5.33$ (m, 2H), 6.87 (d, $J=6.6 \mathrm{~Hz}, 1 \mathrm{H}$ ); IR (KBr) 2905, 1784, 1644, 1426, 1269, 1055, 992, $756 \mathrm{~cm}^{-1}$; HRMS $m / z$ Calcd for $\mathrm{C}_{9} \mathrm{H}_{11} \mathrm{NO}_{4}\left(\mathrm{M}^{+}\right)$: 197.0689. Found: 197.0668.

Electrochemical acetoxylation of 2,3-didehydro- and 1,2-didehydropiperidine derivatives 10a-d, 4, 19 and 23

A typical procedure is exemplified by the anodic oxidation of $\mathbf{4}$. Into a glass beaker (15 $\mathrm{mL}$ ) equipped with two Pt plate electrodes $(10 \mathrm{~mm} \times 20 \mathrm{~mm})$ was added a solution of 4 (0.243g, 1mmol) and AcOK (1.00 g, $10 \mathrm{mmol})$ in acetic acid $(10 \mathrm{~mL})$. After $15 \mathrm{~F} / \mathrm{mol}$ of electricity was passed at a constant current of $0.1 \mathrm{~A}$ ( $4 \mathrm{~h}$, terminal voltage: ca $15 \mathrm{~V}$ ) through the solution cooled with water, saturated aqueous $\mathrm{NaHCO}_{3}(20 \mathrm{~mL})$ was added into the reaction mixture. The organic portion was extracted with AcOEt (20 mL x 3) and the combined organic layer was washed with saturated aqueous $\mathrm{NaHCO}_{3}(20 \mathrm{~mL})$. After the extract was dried over $\mathrm{MgSO}_{4}$ and the solvent was removed in vacuo, the residue was chromatographed on silica gel (AcOEt: $n$-hexane $=1: 3$ ) to afford 1,2,3-triacetoxy-5S-acetoxymethyl- $N$-methoxycarbonylpiperidine (5) in 85\% yield.

5: ${ }^{1} \mathrm{H}-\mathrm{NMR}\left(\mathrm{CDCl}_{3}\right) \delta$ 1.91-2.24 (m, 14H), 3.69-3.82 (m, 3H), 4.03-4.39 (m, 2H), 4.45-4.60 (m, 1H), 4.88-5.07 (m, 1H), 5.15-5.38 (m, 1H), 6.64-6.90 (m, 1H); IR (neat) 2952, 1755, 1597, 1447, 1372, 1240, 1044, $776 \mathrm{~cm}^{-1}$; HRMS (EI) $\mathrm{m} / z$ Calcd for $\mathrm{C}_{14} \mathrm{H}_{19} \mathrm{NO}_{8}\left(\mathrm{M}^{+}-\mathrm{AcOH}\right)$ : 329.1111. Found: 329.1111.

1,2,3-Triacetoxy- $N$-methoxycarbonylpiperidine (11a): ${ }^{1} \mathrm{H}-\mathrm{NMR}\left(\mathrm{CDCl}_{3}\right) \delta$ 1.77-2.25 (m, $11 \mathrm{H}), 3.08-3.17(\mathrm{~m}, 1 \mathrm{H}), 3.74$ and $3.76(2 \mathrm{~s}, 3 \mathrm{H}), 3.95-4.14(\mathrm{~m}, 1 \mathrm{H}), 4.82-5.02$ and 5.14-5.28 (2m, 2H), 6.56-6.78 and 6.93-7.08 (2m, 1H); IR (neat) 2980, 1786, 1420, 1375, 1256, 1051, $764 \mathrm{~cm}^{-1}$; Anal. Calcd for $\mathrm{C}_{13} \mathrm{H}_{19} \mathrm{NO}_{8}$ : C, 49.21; H, 6.04; N, 4.41. Found: C, 49.14; H, 6.22; N, 4.35 .

1,2,3-Triacetoxy- $N$-benzyloxycarbonylpiperidine (11b): ${ }^{1} \mathrm{H}-\mathrm{NMR}\left(\mathrm{CDCl}_{3}\right) \delta$ 1.75-2.24 (m, $11 \mathrm{H}), 3.09-3.27(\mathrm{~m}, 1 \mathrm{H}), 3.97-4.26(\mathrm{~m}, 1 \mathrm{H}), 4.95-5.31(\mathrm{~m}, 4 \mathrm{H}), 6.80$ and $7.10(2 \mathrm{~d}, J=1.0$ and $4.0 \mathrm{~Hz}, 1 \mathrm{H}), 7.35$ (s, 5H); IR (neat) 2953, 1748, 1717, 1370, 1215, 1053, $698 \mathrm{~cm}^{-1}$; HRMS (EI) $m / z$ Calcd for $\mathrm{C}_{19} \mathrm{H}_{23} \mathrm{NO}_{8}\left(\mathrm{M}^{+}\right)$: 393.1424. Found: 393.1464 .

1,2,3-Triacetoxy- $\boldsymbol{N}$-formylpiperidine (11c): ${ }^{1} \mathrm{H}-\mathrm{NMR} \quad\left(\mathrm{CDCl}_{3}\right) \quad \delta \quad 1.80-2.29 \quad(\mathrm{~m}, 11 \mathrm{H})$, 
2.81-3.17 (m, 1H), 4.15-4.46 (m, 1H), 4.91-5.08 (m, 1H), 5.22-5.37 (m, 1H), 5.95, 6.04, 6.35 and 6.43 (4d, $J=0.8,1.0,3.0$ and $4.0 \mathrm{~Hz}, 1 \mathrm{H}$ ), 8.25 and 8.28 (2s, $1 \mathrm{H}$ ); IR (neat) 3567, 2942, 1759, 1698, 1433, 1374, 1256, 1053, $704 \mathrm{~cm}^{-1}$; HRMS (EI) $\mathrm{m} / z$ Calcd for $\mathrm{C}_{12} \mathrm{H}_{17} \mathrm{NO}_{7}\left(\mathrm{M}^{+}\right)$: 287.1005. Found: 287.0981.

1,2,3-Triacetoxy- $N$-benzoylpiperidine (11d): ${ }^{1} \mathrm{H}-\mathrm{NMR}\left(\mathrm{CDCl}_{3}\right) \delta$ 1.84-2.38 (m, $\left.11 \mathrm{H}\right)$, 3.10-3.49 (m, 1H), 4.18-4.59 (m, 1H), 4.92-5.13 (m, 1H), 5.21-5.41 (m, 1H), 6.15-6.44 and 6.61-6.88 (2m, 1H), 7.24-7.51 (m, 5H); IR (neat) 3063, 2940, 1755, 1659, 1374, 1252, 1057, $702 \mathrm{~cm}^{-1}$; Anal. Calcd for $\mathrm{C}_{18} \mathrm{H}_{21} \mathrm{NO}_{7}$ : C, 59.50; H, 5.83; N, 3.85. Found: C, 59.23; H, 6.23; N, 3.65.

(3R,4R,6S)-2,3,4-Triacetoxy-1-aza-8-oxabicyclo[4.3.0]nonan-9-one (20): ${ }^{1} \mathrm{H}-\mathrm{NMR}\left(\mathrm{CDCl}_{3}\right)$ $\delta 1.94(\mathrm{td}, J=12.0$ and $1.8 \mathrm{~Hz}, 1 \mathrm{H}), 2.05-2.18$ (m, $10 \mathrm{H}), 4.02$ (dd, $J=8.6$ and $6.6 \mathrm{~Hz}, 1 \mathrm{H}$ ), 4.20-4.30 (m, 1H), 4.52-4.58 (m, 1H), 5.06-5.10 (m, 2H), 6.31 and 6.59 (2d, $J=1.0$ and 1.8 $\mathrm{Hz}, 3 / 4 \mathrm{H}$ and 1/4H); IR (neat) 2940, 1782, 1420, 1374, 1285, 1048, $764 \mathrm{~cm}^{-1}$; HRMS (EI) $\mathrm{m} / z$ Calcd for $\mathrm{C}_{11} \mathrm{H}_{13} \mathrm{NO}_{6}\left(\mathrm{M}^{+}-\mathrm{AcOH}\right)$ : 255.0743. Found: 255.0726.

\section{Reduction of 1,2,3-triacetoxy- $N$-acylpiperidine derivatives 5, 11a-d, and 20}

A typical procedure is exemplified by the reduction of $\mathbf{5}$. Into a solution of 5 (0.389 g, 1 mmol) and $\mathrm{Et}_{3} \mathrm{SiH}(0.140 \mathrm{~g}, 1.2 \mathrm{mmol})$ in $\mathrm{CH}_{2} \mathrm{Cl}_{2}(3 \mathrm{~mL})$ was added methanesulfonic acid $(0.144 \mathrm{~g}, 1.5 \mathrm{mmol})$ at $0{ }^{\circ} \mathrm{C}$. After stirring for $10 \mathrm{~min}$, into a mixture of AcOEt (20 mL) and saturated aqueous $\mathrm{NaHCO}_{3}(20 \mathrm{~mL})$ was poured the reaction mixture. The organic portion was extracted with AcOEt (20 mL x 3) and the combined organic layer was washed with saturated aqueous $\mathrm{NaHCO}_{3}(20 \mathrm{~mL})$. After the extract was dried over $\mathrm{MgSO}_{4}$ and the solvent was removed in vacuo, the residue was chromatographed on silica gel (AcOEt: $n$-hexane = 1:2) to afford 2,3-diacetoxy-5S-acetoxymethyl- $N$-methoxycarbonylpiperidine (6) in $62 \%$ yield as a mixture of stereoisomers. Recrystalization of $\mathbf{6}$ from AcOEt and $n$-hexane afforded $2 S, 3 S, 5 S$-isomer.

6 $_{2 S, 3 S, 5 s}:[\alpha]^{26}+40.0\left(c\right.$ 0.5, $\left.\mathrm{CHCl}_{3}\right)$; mp 102-104 ${ }^{\circ} \mathrm{C} ;{ }^{1} \mathrm{H}-\mathrm{NMR}\left(\mathrm{CDCl}_{3}\right) \delta$ 1.77-1.87 (m, 1H), 2.04 (s, 3H), 2.06 (s, 3H), 2.10 (s, 3H), 2.10-2.23 (m, 1H), 3.34 (d, J = $15.0 \mathrm{~Hz}, 1 \mathrm{H}$ ), 3.71 (s, 3H), 4.13 (dd, $J=11.3$ and $5.9 \mathrm{~Hz}, 1 \mathrm{H}), 4.23$ (d, $J=15.0 \mathrm{~Hz}, 1 \mathrm{H}), 4.40$ (t, $J=9.7 \mathrm{~Hz}, 1 \mathrm{H}$ ), 4.54-4.70 (m, 1H), 4.76-4.87 (m, 1H), 4.91-4.99 (m, 1H); IR (KBr) 2959, 1750, 1701, 1441, 
1374, 1223,1069, $772 \mathrm{~cm}^{-1}$; Anal. Calcd for $\mathrm{C}_{14} \mathrm{H}_{21} \mathrm{NO}_{8}$ : C, 50.75; H, 6.39; N, 4.23. Found: C, 50.88; H, 6.68; N, 4.26. Major isomer of 6 was detected by HPLC method; YMC-Pack SIL (0.46 cmø x $15 \mathrm{~cm}), n$-hexane/ethanol = 10:1, wavelength: $210 \mathrm{~nm}$, flow rate: $0.5 \mathrm{~mL} / \mathrm{min}$, retention time:11.4 min.

2,3-Diacetoxy- $N$-methoxycarbonylpiperidine (12a): ${ }^{1} \mathrm{H}-\mathrm{NMR}\left(\mathrm{CDCl}_{3}\right) \delta 1.86-2.19(\mathrm{~m}, 8 \mathrm{H})$, 3.20-3.50 (m, 2H), 3.70 (s, 3H), 3.77-3.98 (m, 1H), 4.71-4.87 (m, 1H), 4.88-4.98 (m, 1H), 4.99-5.13 (m, 1H); IR (neat) 2959, 1755, 1471, 1374, 1057, $770 \mathrm{~cm}^{-1}$; HRMS m/z Calcd for $\mathrm{C}_{11} \mathrm{H}_{17} \mathrm{NO}_{6}\left(\mathrm{M}^{+}\right)$: 259.1055. Found: 259.1042. Diastereomer ratio of 12a was determined by HPLC method; YMC-Pack SIL (0.46 cmø x $15 \mathrm{~cm})$, $n$-hexane/ethanol = 10:1, wavelength: $210 \mathrm{~nm}$, flow rate: $0.5 \mathrm{~mL} / \mathrm{min}$, retention time: $8.2 \mathrm{~min}$ for trans-isomer, $9.1 \mathrm{~min}$ for cis-isomer.

2,3-Diacetoxy- $N$-benzyloxycarbonylpiperidine (12b): ${ }^{1} \mathrm{H}-\mathrm{NMR}\left(\mathrm{CDCl}_{3}\right) \delta$ 1.90-2.12 (m, 8H), 3.30-4.05 (m , 4H), 4.18-5.12 (m, 4H), 7.35 (s, 5H); IR (neat) 3033, 2942, 1752, 1433, 1254, 1055, 766, $700 \mathrm{~cm}^{-1}$; HRMS $\mathrm{m} / z$ Calcd for $\mathrm{C}_{17} \mathrm{H}_{21} \mathrm{NO}_{6}\left(\mathrm{M}^{+}\right)$: 335.1369. Found: 335.1349. Diastereomer ratio of $\mathbf{1 2 b}$ was determined by HPLC method; YMC-Pack SIL (0.46 cmø x $15 \mathrm{~cm}$ ), $n$-hexane/ethanol = 15:1, wavelength: $210 \mathrm{~nm}$, flow rate: $0.5 \mathrm{~mL} / \mathrm{min}$, retention time: $9.3 \mathrm{~min}$ for trans-isomer, $10.4 \mathrm{~min}$ for $\mathrm{cis}$-isomer.

2,3-Diacetoxy- $\boldsymbol{N}$-formylpiperidine (12c): ${ }^{1} \mathrm{H}-\mathrm{NMR}\left(\mathrm{CDCl}_{3}\right) \delta$ 1.80-2.08 (m, 8H), 3.15-3.75 and 3.95-4.35 (2m, 4H), 4.75-4.88 and 4.95-5.45 (2m, 2H), 7.95, 7.97, 8.08, and 8.10 (4s, 1H); IR (neat) 3650, 2940, 1759, 1690, 1439, 1372, 1260, $1046 \mathrm{~cm}^{-1}$; HRMS m/z Calcd for $\mathrm{C}_{10} \mathrm{H}_{15} \mathrm{NO}_{5}\left(\mathrm{M}^{+}\right)$: 229.0950. Found: 229.0975. Diastereomer ratio of 12c was determined by HPLC method; YMC-Pack SIL (0.46 cmø x $15 \mathrm{~cm})$, $n$-hexane/ethanol = 10:1, wavelength: $210 \mathrm{~nm}$, flow rate: $0.5 \mathrm{~mL} / \mathrm{min}$, retention time: $9.0 \mathrm{~min}$ for trans-isomer, $9.7 \mathrm{~min}$ for cis-isomer.

2,3-Diacetoxy- $\boldsymbol{N}$-benzoylpiperidine (12d): ${ }^{1} \mathrm{H}-\mathrm{NMR}\left(\mathrm{CDCl}_{3}\right) \delta 1.70-2.20$ (m, 8H), 3.20-4.40 (m, 4H), 4.68-5.22 (m, 2H), 7.41 (s, 5H); IR (neat) 2940, 1744, 1640, 1431, 1372, 1248, 706 $\mathrm{cm}^{-1}$; HRMS $m / z$ Calcd for $\mathrm{C}_{16} \mathrm{H}_{19} \mathrm{NO}_{5}\left(\mathrm{M}^{+}\right)$: 305.1263. Found: 305.1273. Diastereomer ratio of 12d was determined by HPLC method; YMC-Pack SIL (0.46 cmø x 15 cm), $n$-hexane/ethanol $=10: 1$, wavelength: $210 \mathrm{~nm}$, flow rate: $0.5 \mathrm{~mL} / \mathrm{min}$, retention time: 25.9 
min for trans-isomer, 29.5 min for cis-isomer.

(3R,4R,6S)-3,4-Diacetoxy-1-aza-8-oxabicyclo[4.3.0]nonan-9-one (21): $[\alpha]^{26}-75.2$ (c 0.6, $\mathrm{CHCl}_{3}$ ); mp $127-129^{\circ} \mathrm{C}$ (from AcOEt and n-hexane), (uncorrected); ${ }^{1} \mathrm{H}-\mathrm{NMR}\left(\mathrm{CDCl}_{3}\right) \delta$ 1.90-2.05 (m, 2H), 2.09 (s, 3H), 2.13 (s, 3H), 3.33 (dd, $J=15.0$ and 2.1 Hz, 1H), 3.92-4.05 (m, 3H), 4.38-4.48 (m, 1H), 4.80-4.85 (m, 1H), 5.08-5.12 (m, 1H); IR (neat) 2932, 1744, 1422, 1372, 1221, 1061, 914, $768 \mathrm{~cm}^{-1}$; Anal. Calcd for $\mathrm{C}_{11} \mathrm{H}_{15} \mathrm{NO}_{6}$ : C, 51.36; H, 5.88; N, 5.45. Found: C, 51.49; H, 6.08; N, 5.44. Major isomer of 21 was detected by HPLC method; YMC-Pack SIL (0.46 cmø x $15 \mathrm{~cm}), n$-hexane/ethanol = 5:1, wavelength: $210 \mathrm{~nm}$, flow rate: $0.5 \mathrm{~mL} / \mathrm{min}$, retention time: $18.9 \mathrm{~min}$.

\section{Preparation of 2,3-didehydropiperidine derivative 33.}

Into a round-bottomed flask (25 $\mathrm{mL}$ ) equipped with a magnetic stirrer and containg 19 (0.423 g, $2.5 \mathrm{mmol})$ in acetic acid $(10 \mathrm{~mL})$ was added $\mathrm{NaBH}_{4}(0.946 \mathrm{~g}, 10 \mathrm{mmol})$. The reaction vessel was cooled with water. After stirring for $10 \mathrm{~min}$, water $(10 \mathrm{~mL})$ was added slowly to the reaction solution at $0{ }^{\circ} \mathrm{C}$. The mixture was extracted with AcOEt (20 mL x 3). The combined extracts were washed with saturated aqueous $\mathrm{NaHCO}_{3}(20 \mathrm{~mL})$. After the extracts were dried over anhydrous $\mathrm{MgSO}_{4}$, filtered, and concentrated in vacuo, the residue was chromatographed on silica gel (AcOEt: $n$-hexane $=1: 2$ ) to afford 33 in $70 \%$ yield.

6S-1-Aza-3,4-didehydro-8-oxabicyclo[4.3.0]nonan-9-one (33): $[\alpha]_{D}^{30}-166.9$ (c 1.0, $\left.\mathrm{CHCl}_{3}\right) ;{ }^{1} \mathrm{H}-\mathrm{NMR}\left(\mathrm{CDCl}_{3}\right) \delta$ 2.11-2.37 (m, 2H), 3.64-3.75 (m, 1H), 3.76-3.89 (m, 1H), 4.03 (dd, $J=8.7 \mathrm{~Hz}$ and $5.7 \mathrm{~Hz}, 1 \mathrm{H}), 4.08-4.14$ and 4.16-4.21 (2m, 1H), 4.52 (t, $J=8.3 \mathrm{~Hz}, 1 \mathrm{H})$, 5.70-5.89 (m, 2H); IR (neat) 2977, 1777, 1457, 1242, 1208, 1078, 961, $764 \mathrm{~cm}^{-1}$; HRMS (EI) $m / z$ Calcd for $\mathrm{C}_{7} \mathrm{H}_{9} \mathrm{NO}_{2}\left(\mathrm{M}^{+}\right)$: 139.0633, Found: 139.0609 .

Osmium oxidation of 2,3-didehydropiperidine derivatives 4, 19, 10a, and 33 and the successive acetoxylations.

A typical procedure is exemplified by the osmium oxidation of 10a. Into a round-bottomed flask (25 mL) equipped with a magnetic stirrer was added a solution of 5 (0.171 g, $1 \mathrm{mmol}$ ) and NMO (50\% in water, $0.351 \mathrm{~g}, 1.5 \mathrm{mmol})$ in acetone $(0.5 \mathrm{~mL})$ and $\mathrm{H}_{2} \mathrm{O}$ $(2.5 \mathrm{~mL})$. To a stirred solution at room temperature was added osmium tetraoxide (4wt \% solution in water, 2 drops, $0.01 \mathrm{mmol}$ ). After the mixture was stirred overnight at room 
temperature, $10 \%$ aqueous $\mathrm{Na}_{2} \mathrm{~S}_{2} \mathrm{O}_{3}(5 \mathrm{~mL})$ was added into the reaction mixture. The resulting mixture was concentrated under reduced pressure. Pyridine (2 $\mathrm{mL})$ and acetic anhydride (2 $\mathrm{mL}$ ) were then added to the residue and the mixture stirred at room temperature for $2 \mathrm{~h}$. The mixture was concentrated under reduced pressure. To the residue was added water $(10 \mathrm{~mL})$ and the organic portion was extracted with AcOEt (20 mL x 3). The combined extracts were dried over anhydrous $\mathrm{MgSO}_{4}$, filtered, and concentrated in vacuo. The residue was chromatographed on silica gel (AcOEt: $n$-hexane $=1: 5$ ) to afford 1,2,3-triacetoxy- $N$-methoxycarbonylpiperidine (11a) in 71\% yield.

11a: ${ }^{1} \mathrm{H}-\mathrm{NMR}\left(\mathrm{CDCl}_{3}\right) \delta$ 1.78-1.88 (m, 1H), 1.92-2.05 (m, 1H), 2.01, 2.10 and $2.11(3 \mathrm{~s}, 9 \mathrm{H})$, 3.09-3.23 (m, 1H), 3.76 (s, 3H), 4.06-4.29 (m, 1H), 5.18-5.28 (m, 2H), 6.71 (br s, 1H); IR (neat) 2959, 1748, 1449, 1372, 1223, 1057, $772 \mathrm{~cm}^{-1}$; HRMS (EI) $\mathrm{m} / z$ Calcd for $\mathrm{C}_{13} \mathrm{H}_{19} \mathrm{NO}_{8}$ $\left(\mathrm{M}^{+}\right)$: 317.1111. Found: 317.1116.

By similar procedures as above, 4 was converted into a mixture of 2,3-diacetoxy-5S-acetoxymethyl-1-methoxy- $N$-methoxycarbonylpiperidine (29a) and 1,2,3-triacetoxy-5S-acetoxymethyl- $N$-methoxycarbonylpiperidine $(29 b)$ was obtained in 77\% yield (29a:29b $=0.4: 0.6)$. ${ }^{1} \mathrm{H}-\mathrm{NMR}\left(\mathrm{CDCl}_{3}\right) \delta$ 1.85-1.95 (m, 2H), 2.02, 2.03, 2.06, 2.07, 2.096, 2.100, 2.12 (7s, 10.8H), 3.34 and $3.37(2 \mathrm{~s}, 1.2 \mathrm{H}), 3.75$ and $3.77(2 \mathrm{~s}, 3 \mathrm{H}), 4.07-4.20(\mathrm{~m}, 1 \mathrm{H}), 4.22-4.41(\mathrm{~m}, 1 \mathrm{H})$, 4.54-4.79 (m, 1H), 5.18-5.52 (m, 2H), 5.72-5.84 (m, 0.4H), 6.70-6.90 (m, 0.6H); IR (neat) 2959, 1744, 1445, 1370, 1225, 1090, $774 \mathrm{~cm}^{-1}$.

(3R,4S,6S)-3,4-Diacetoxy-2-methoxy-1-aza-8-oxabicyclo[4.3.0]nonan-9-one (32) (85\% yield from 19): ${ }^{1} \mathrm{H}-\mathrm{NMR}\left(\mathrm{CDCl}_{3}\right) \delta$ 1.84-2.02 (m, 2H), 2.04 (s, 3H), 2.11 (s, 3H), $3.38(\mathrm{~s}, 3 \mathrm{H})$, 3.98-4.10 (m, 2H), 4.48-4.56 (m, 1H), 5.01 (d, $J=2.4$ Hz, 1H), 5.19-5.29 (m, 2H); IR (neat) 2940, 1771, 1414, 1374, 1238, 1102, 970, $764 \mathrm{~cm}^{-1}$; HRMS (EI) $\mathrm{m} / z$ Calcd for $\mathrm{C}_{12} \mathrm{H}_{17} \mathrm{NO}_{7}$ $\left(\mathrm{M}^{+}\right)$: 287.1005. Found: 287.1014.

Using similar oxidation procedure, $\mathbf{3 3}$ was successively oxidized and acetoxylated to afford a mixture of $(3 S, 4 R, 6 S)$-3,4-diacetoxy-1-aza-8-oxabicyclo[4.3.0]nonan-9-one $\left(\mathbf{2 8}_{2 S, 3 R, 5 S}\right)$ and $(3 R, 4 S, 6 S)$-isomer $\quad\left(\mathbf{2 8}_{2 R, 3 S, 5 S}\right) \quad\left(\mathbf{2 8}_{2 S, 3 R, 5 S}: \mathbf{2 8}_{2 R, 3 S, 5 S}=76: 24\right)$ in $80 \%$ yield. (3S,4R,6S)-3,4-Diacetoxy-1-aza-8-oxabicyclo[4.3.0]nonan-9-one $\left(\mathbf{2 8}_{2 S, 3 R, 5 S}\right):[\alpha]_{\mathrm{D}}^{28}-53.3$ (c 1.5, $\mathrm{CHCl}_{3}$ ), (containing $4 \%$ of $(3 R, 4 S, 6 S)$-isomer $\mathbf{2 8}_{2 S, 3 R, 5 s)}$; ${ }^{1} \mathrm{H}-\mathrm{NMR}\left(\mathrm{CDCl}_{3}\right) \delta 1.70-1.83$ (m, 1H), 2.03 (s, 3H), 2.07-2.13 (m, 1H), 2.14 (s, 3H), 3.22 (t, $J=12.0$ Hz, 1H), 3.89-4.09 (m, 
3H), 4.46 (t, $J=9.3 \mathrm{~Hz}, 1 \mathrm{H}), 4.80-4.90$ (m, 1H), 5.50 (br s, 1H); IR (neat) 2940, 1781, 1485, 1375, 1266, 1177, 1071, 974, $762 \mathrm{~cm}^{-1}$; HRMS (EI) $m / z$ Calcd for $\mathrm{C}_{11} \mathrm{H}_{15} \mathrm{NO}_{6}\left(\mathrm{M}^{+}\right)$: 257.0899. Found: 257.0892.

\section{Reduction of $\alpha$-alkoxyl group of 11a, 29a, 29b and 32.}

A typical procedure is exemplified by the reduction of 11a. To $1 \mathrm{mmol}$ of 11a was added $\mathrm{Et}_{3} \mathrm{SiH}$ (0.174 g, $\left.1.5 \mathrm{mmol}\right)$ in $\mathrm{CH}_{2} \mathrm{Cl}_{2}$ (3 mL), methanesulfonic acid (0. $192 \mathrm{~g}, 2.0 \mathrm{mmol}$ ) was then added at $0{ }^{\circ} \mathrm{C}$. After stirring for $10 \mathrm{~min}$, into a mixture of AcOEt (20 mL) and saturated aqueous $\mathrm{NaHCO}_{3}(20 \mathrm{~mL})$ was poured the reaction mixture. The organic portion was extracted with AcOEt (20 mL x 3) and the combined organic layers were washed with saturated aqueous $\mathrm{NaHCO}_{3}(20 \mathrm{~mL})$. After the extracts were dried over anhydrous $\mathrm{MgSO}_{4}$, filtered, and concentrated in vacuo, the residue was chromatographed on silica gel (AcOEt: $n$-hexane $=1: 5)$ to afford 2,3-diacetoxy- $N$-methoxycarbonylpiperidine (12a) in 70\% yield. cis-2,3-Diacetoxy- $N$-methoxycarbonylpiperidine (12acis): ${ }^{1} \mathrm{H}-\mathrm{NMR} \quad\left(\mathrm{CDCl}_{3}\right) \delta$ 1.72-1.83 (m, 1H), 1.87-2.02 (m, 1H), 2.07 and 2.08 (2s, 6H), 3.20-3.48 (m, 2H), 3.70 (s, 3H), 3.87 and 3.91 (2d, $J=6.0$ and $6.0 \mathrm{~Hz}, 2 \mathrm{H}$ ), 4.98-5.13 (m, 2H); IR (neat) 2959, 1755, 1474, 1372, 1278, 1057, $770 \mathrm{~cm}^{-1}$; HRMS (EI) $\mathrm{m} / z$ Calcd for $\mathrm{C}_{11} \mathrm{H}_{17} \mathrm{NO}_{6}\left(\mathrm{M}^{+}\right)$: 259.1056. Found: 259.1049.

2S,3R-Diacetoxy-5S-acetoxymethyl- $N$-methoxycarbonylpiperidine $\left(27_{2 S, 3 R, 5 S}\right)(70 \%$ yield from a mixture of 29a and 29b): $[\alpha]^{30}{ }_{\mathrm{D}}+37.0\left(c 1.0, \mathrm{CHCl}_{3}\right) ;{ }^{1} \mathrm{H}-\mathrm{NMR}\left(\mathrm{CDCl}_{3}\right) \delta 1.75$ and 1.78 (2d, $J=4.4 \mathrm{~Hz}, 1 \mathrm{H}), 2.02$ and 2.07 and 2.08 (3s, 9H), 2.09-2.11 (m, 1H), 3.19 (d, $J=15.0$ Hz, 1H), 3.72 (s, 3H), 4.10 and 4.15 (2d, $J=5.7$ Hz, 1H), 4.23-4.38 (m, 2H), 4.69-4.82 (br s, 1H), 5.03-5.13 (m, 1H), 5.19 (br s, 1H); IR (neat) 2959, 1755, 1709, 1451, 1374, 1256, 1055, $770 \mathrm{~cm}^{-1}$; HRMS $m / z$ Calcd for $\mathrm{C}_{14} \mathrm{H}_{21} \mathrm{NO}_{8}\left(\mathrm{M}^{+}\right)$: 331.1267. Found: 331.1258. Major isomer of 27 was detected by HPLC method; YMC-Pack SIL (0.46 cmø x $15 \mathrm{~cm}), n$-hexane/ethanol = 10:1, wavelength: $210 \mathrm{~nm}$, flow rate: $0.5 \mathrm{~mL} / \mathrm{min}$, retention time: $12.2 \mathrm{~min}$.

(3R,4S,6S)-3,4-Diacetoxy-1-aza-8-oxabicyclo[4.3.0]nonan-9-one $\quad\left(28_{2 R, 3 S, 5 S}\right) \quad(81 \%$ yield from 32): $[\alpha]_{\mathrm{D}}^{29}-48.0$ (c $0.5, \mathrm{CHCl}_{3}$ ); $\mathrm{mp} 122-123^{\circ} \mathrm{C}$ (from AcOEt and $n$-hexane), (uncorrected); ${ }^{1} \mathrm{H}-\mathrm{NMR}\left(\mathrm{CDCl}_{3}\right) \delta$ 1.89-1.99 (m, 2H), 2.05 (s, 3H), 2.11 (s, 3H), 3.13 (dd, $J=$ 12.8 and $1.8 \mathrm{~Hz}, 1 \mathrm{H}), 3.84-3.95(\mathrm{~m}, 1 \mathrm{H}), 4.04$ (dd, $J=8.4$ and $3.3 \mathrm{~Hz}, 1 \mathrm{H}), 4.10$ (d, $J=12.5$ Hz, 1H), 4.44 (t, $J=7.8 \mathrm{~Hz}, 1 \mathrm{H}), 4.92-5.03$ (m, 1H), 5.19 (br s, 1H); IR (KBr) 2936, 1763, 
1431, 1374, 1258, 1073, 986, $764 \mathrm{~cm}^{-1}$; Anal. Calcd for $\mathrm{C}_{11} \mathrm{H}_{15} \mathrm{NO}_{6}$ : C, 51.36; H, 5.88; N, 5.45. Found: C, 51.43; H, 5.93; N, 5.40. Major isomer of 11 was detected by HPLC method; YMC-Pack SIL (0.46 cmø x $15 \mathrm{~cm})$, $n$-hexane/ethanol = 5:1, wavelength: 210nm, flow rate: $0.5 \mathrm{~mL} / \mathrm{min}$, retention time: $21.6 \mathrm{~min}$.

\section{Synthesis of $5 S$-acetoxymethyl-2S,3R-dihydroxy- $N$-methoxycarbonylpiperidine} $\left(30_{2 S, 3 R, 5 S}\right)$ and successive tosylation.

Into a round-bottomed flask $(25 \mathrm{~mL})$ equipped with a magnetic stirrer and containing a solution of 4 (0.243 g, $1 \mathrm{mmol})$ in acetone $(0.5 \mathrm{~mL})$ and $\mathrm{H}_{2} \mathrm{O}(2.5 \mathrm{~mL})$ was added NMO (50\% in water, $0.351 \mathrm{~g}, 1.5 \mathrm{mmol})$. To a stirred solution at room temperature was added osmium tetraoxide (4wt \% solution in water, 2drops, $0.01 \mathrm{mmol}$ ). After the mixture was stirred overnight at room temperature, $10 \%$ aqueous $\mathrm{Na}_{2} \mathrm{~S}_{2} \mathrm{O}_{3}(5 \mathrm{~mL})$ was added into the reaction mixture. The resulting mixture was concentrated under reduced pressure and to the residue was added water $(1 \mathrm{~mL})$. The organic portion was extracted with AcOEt (15 mL x 8). The combined extracts were dried over anhydrous $\mathrm{MgSO}_{4}$, filtered, and concentrated in vacuo to afford a crude mixture of mixture of $5 S$-acetoxymethyl-1,2,3-trihydroxy- $N$-methoxycarbonylpiperidine $\quad$ and $5 S$-acetoxymethyl-2,3-dihydroxy-1-methoxy- $N$-methoxycarbonylpiperidine (0.5:0.5) : ${ }^{1} \mathrm{H}-\mathrm{NMR}\left(\mathrm{CDCl}_{3}\right)$ $\delta$ 1.70-1.85 (m, 1H), 1.89-2.04 (m, 1H), 2.06 (s, 3H), 3.33 (s, 1.5H), 3.74 and $3.76(2 \mathrm{~s}, 3 \mathrm{H})$, 3.91-4.08 (m, 1H), 4.10-4.20 (m, 1H), 4.21-4.42 (m, 2H), 4.47-4.75 (m, 1H), 5.35-5.44 and 5.51-5.62 and 5.79-5.84 (3m, 1H); IR (neat) 3413, 2959, 1742, 1449, 1356, 1240, 1086, 774 $\mathrm{cm}^{-1}$.

To the mixture was added $\mathrm{Et}_{3} \mathrm{SiH}(0.174 \mathrm{~g}, 1.5 \mathrm{mmol})$ in $\mathrm{CH}_{2} \mathrm{Cl}_{2}(3 \mathrm{~mL})$ and added methanesulfonic acid $(0.192 \mathrm{~g}, 2.0 \mathrm{mmol})$ at $0{ }^{\circ} \mathrm{C}$. After stirring for $10 \mathrm{~min}$, into a mixture of AcOEt $(20 \mathrm{~mL})$ and saturated aqueous $\mathrm{NaHCO}_{3}(20 \mathrm{~mL})$ was poured the reaction mixture. The organic portion was extracted with AcOEt (20 mL x 3) and the combined organic layers were washed with saturated aqueous $\mathrm{NaHCO}_{3}(20 \mathrm{~mL})$. After the extracts were dried over anhydrous $\mathrm{MgSO}_{4}$, filtered, and concentrated in vacuo, the residue was chromatographed on silica gel (AcOEt: $n$-hexane $=3: 1$ ) to afford $5 S$-acetoxymethyl-2S,3R-dihydroxy- $N$-methoxycarbonylpiperidine $\left(\mathbf{3 0}_{2 S, 3 R, 5 S}\right)$ in $78 \%$ yield from 4. $\left(\mathbf{3 0}_{2 S, 3 R, 5 S}\right)$ : $[\alpha]^{30}-6.0\left(c\right.$ 1.0, $\left.\mathrm{CHCl}_{3}\right)$; ${ }^{1} \mathrm{H}-\mathrm{NMR}\left(\mathrm{CDCl}_{3}\right) \delta 1.73$ and $1.77(2 \mathrm{~d}, J=4.2 \mathrm{~Hz}, 1 \mathrm{H}), 1.91-2.02(\mathrm{~m}, 1 \mathrm{H}), 2.05(\mathrm{~s}, 3 \mathrm{H}), 2.24$ (d, $J=6.5 \mathrm{~Hz}, 1 \mathrm{H}$ ), 2.31-2.48 (br s, 1H), 3.10 (d, $J=15.0 \mathrm{~Hz}, 1 \mathrm{H}$ ), 3.72 (s, 3H), 3.80-3.96 (m, 
2H), 4.06-4.38 (m, 3H), 4.57-4.73 (br s, 1H); IR (neat) 3447, 2959, 1744, 1698, 1456, 1370, 1258, 1140, 1080, $770 \mathrm{~cm}^{-1}$; HRMS $\mathrm{m} / z$ Calcd for $\mathrm{C}_{10} \mathrm{H}_{17} \mathrm{NO}_{6}\left(\mathrm{M}^{+}\right)$: 247.1056. Found: 247.1058 .

To $30_{2 S, 3 R, 5 s}(0.1 \mathrm{~g}, 0.4 \mathrm{mmol})$ was added $p$-toluenesulfonyl chloride (0.381 g, $\left.2 \mathrm{mmol}\right), \mathrm{Et}_{3} \mathrm{~N}$ (0.049 g, $0.48 \mathrm{mmol}$ ), and DMAP $(0.244 \mathrm{~g}, 2 \mathrm{mmol})$ in $\mathrm{CH}_{2} \mathrm{Cl}_{2}(2 \mathrm{~mL})$. After the mixture was stirred for 3 days at room temperature, into a mixture of AcOEt $(20 \mathrm{~mL})$ and saturated aqueous $\mathrm{NaHCO}_{3}(10 \mathrm{~mL})$ was poured the reaction mixture. The organic portion was extracted with AcOEt (20 mL x 3). After the extracts were dried over anhydrous $\mathrm{MgSO}_{4}$, filtered, and concentrated in vacuo, the residue was chromatographed on silica gel (AcOEt: $n$-hexane $=\quad 1: 6)$

to

afford

$5 S$-acetoxymethyl-2S,3R-bis(p-toluenesulfonyloxy)- $N$-methoxycarbonylpiperidine (31 $2 S, 3 R, 5 s)$ in $46 \%$ yield.

31 2 S,3R,5s: $[\alpha]_{\mathrm{D}}^{30}+32.4$ (c 1.0, $\mathrm{CHCl}_{3}$ ); mp 136-139 ${ }^{\circ} \mathrm{C}$ (from AcOEt and $n$-hexane); ${ }^{1} \mathrm{H}-\mathrm{NMR}$ $\left(\mathrm{CDCl}_{3}\right) \delta 1.64$ and 1.71 (2d, $\left.J=3.6 \mathrm{~Hz}, 1 \mathrm{H}\right), 2.01$ (s, 3H), 2.10-2.26 (m, 1H), 2.46 (s, 6H), 3.09 (d, $J=15.3 \mathrm{~Hz}, 1 \mathrm{H}), 3.69$ (s, 3H), 3.97-4.16 (m, 2H ), 4.45 (d, $J=15.3 \mathrm{~Hz}, 1 \mathrm{H}$ ), 4.55-4.72 (m, 3H), 7.30-7.39 (m, 4H), 7.64 (d, $J=8.1 \mathrm{~Hz}, 2 \mathrm{H}), 7.79$ (d, $J=8.4 \mathrm{~Hz}, 2 \mathrm{H})$; IR (KBr) 2957, 1748, 1701, 1449, 1364, 1246, 1140, 1124, 918, $770 \mathrm{~cm}^{-1}$; Anal. Calcd for $\mathrm{C}_{24} \mathrm{H}_{29} \mathrm{NO}_{10} \mathrm{~S}_{2}$ : C, 51.88; H, 5.26; N, 2.52. Found: C, 51.92; H, 5.39; N, 2.52.

\section{References and Notes}

1. Recent representative reviews: (a) Heightman, T. D.; Vasella, A. T. Angew. Chem. Int. Ed. 1999, 38, 750-770; (b) Butters, T. D.; Dwek, R. A.; Platt, F. M. Chem. Rev. 2000, 100, 4683-4696; (c) Afarinkia, K.; Bahar, A. Tetrahedron: Asymmetry 2005, 16, 1239-1287; (d) Huang, P.-Q. Synlett 2006, 1133-1149;

2. Recent representative literatures: (a) Asano, K.; Hakogi, T.; Iwama, S.; Katsumura, S. Chem. Commun. 1999, 41-42; (b) Sawada, D.; Takahashi, H.; Ikegami, S. Tetrahedron Lett. 2003, 44, 3085-3088; (c) Moriyama, H.; Tsukida, T.; Inoue, Y.; Yokota, K.; Yoshino, K.; Kondo, H.; Miura, N.; Nishimura, S. J. Med. Chem. 2004, 47, 1930-1938; (d) Felpin, F.-X.; Bouberkeur, K.; Lebrenton, J. J. Org. Chem. 2004, 69, 1497-1503; (e) Kato, A.; Kato, N.; Kano, E.; Adachi, I.; Ikeda, K.; Yu, L.; Okamoto, T.; Banba, Y.; Ouchi, H.; Takahata, H.; Asano, N.; J. Med. Chem. 2005, 48, 2036-2044; (f) Boglio, C.; Stahlke, S.; Thorimbert, S.; Malacria, M. Org. Lett. 2005, 7, 4851-4854; (g) Calderón, F.; Doyagüez, 
E. G.; Fernández-Mayoralas, A. J. Org. Chem. 2006, 71, 6258-6261; (h) Song, X.; Hollingsworth, R. I. Tetrahedron Lett. 2007, 48, 3115-3118; (i) Yokoyama, H.; Kobayashi, H.; Miyazawa, M.; Yamaguchi, S.; Hirai, Y. Heterocycles 2007, 74, 283-292; (j) Ruiz, M.; Ruanova, T. M.; Blanco, O.; Núñez, F.; Pato, C.; Ojea, V. J. Org. Chem. 2008, 73, 2240-2255; (k) Pandey, G.; Bharadwaj, K. C.; Khan, M. I.; Shashidhara, K. S.; Puranik, V. G. Org. Biomol. Chem. 2008, 6, 2587-2595. (l) Ferreira, F.; Botuha, C.; Chemla, F.; Pérez-Luna, A. J. Org. Chem. 2009, 74, 2238-2241. (m) Aravind, A.; Sankar, M. G.; Varghese, B.; Baskaran, S. J. Org. Chem. 2009, 74, 2858-2861.

3. Andersen, S. M.; Ekhart, C.; Lundt, I.; Stütz, A. E. Carbohydr. Res. 2000, 326, 22-33.

4. Lemaire, M.; Veny, N.; Gefflaut, T.; Gallienne, E.; Chênevert, R.; Bolte, J. Synlett 2002, 1359-1361.

5. Furukubo, S.; Moriyama, N.; Onomura, O.; Matsumura, Y. Tetrahedron Lett. 2004, 45, 8177-8181.

6. (a) Shono, T.; Hamaguchi, H.; Matsumura, Y. J. Am. Chem. Soc. 1975, 97, 4264-4268; (b) Nyberg, K.; Servin, R. Acta Chem. Scand. Ser. B 1976, 30, 640-642; (c) Shono, T.; Matsumura, Y.; Kanazawa, T.; Habuka, M.; Uchida, K.; Toyoda, K. J. Chem. Res. (M); 1984; 2876-2889.

7. (a) Nyberg, K. Synthesis 1976, 545-546. (b) Shono, T.; Matsumura, Y.; Tsubata, T.; Sugihara, Y.; Yamane, S.-I.; Kanazawa, T.; Aoki, T. J. Am. Chem. Soc. 1982, 104, 6697-6703; (c) Kim, S.; Yoon, J.-Y. Synthesis 2000, 1622-1630; (d) Okitsu, O.; Suzuki, R.; Kobayashi, S. J. Org. Chem. 2001, 66, 809-823.

8. Shono, T.; Matsumura, Y.; Onomura, O.; Ogaki, M.; Kanazawa, T. J. Org. Chem. 1987, 52, 536-541.

9. (a) Shono, T.; Matsumura, Y.; Onomura, O.; Yamada, Y. Tetrahedron Lett. 1987, 28, 4073-4074; (b) Matsumura, Y.; Kanda, Y.; Shirai, K.; Onomura, O.; Maki, T. Tetrahedron 2000, 56, 7411-7422; (c) Onomura, O.; Kanda, Y.; Nakamura, Y.; Maki, T.; Matsumura, Y. Tetrahedron Lett. 2002, 43, 3229-3231; (d) Kanda, Y.; Onomura, O.; Maki, T.; Matsumura, Y. Chirality 2003, 44, 89-94; (e) Matsumura, Y.; Onomura, O.; Suzuki, H.; Furukubo, S.; Maki, T.; Li, C.-J. Tetrahedron Lett. 2003, 44, 5519-5522; (f) Onomura, O.; Kanda, Y.; Imai, M.; Matsumura, Y. Electrochim. Acta 2005, 50, 4926-4935; (g) Minato, D.; Imai, M.; Kanda, Y.; Onomura, O.; Matsumura, Y. Tetrahedron Lett. 2006, 47, 5485-5488; (h) Matsumura, Y.; Minato, D.; Onomura, O. J. Organomet. Chem. 2007, 692, 654-663; (i) Onomura, O.; Fujimura, N.; Oda, T.; Matsumura, Y.; Demizu, Y. Heterocycles 2008, 76, 177-182. 
10. (a) Shono, T.; Matsumura, Y.; Onomura, O.; Kanazawa, T.; Habuka, M. Chem. Lett. 1984, 1101-1104; (b) Shono, T.; Matsumura, Y.; Onomura, O.; Sato, M. J. Org. Chem. 1988, 53, 4118-4121; (c) Libendi, S. S.; Ogino, T.; Onomura, O.; Matsumura, Y. J. Electrochem. Soc. 2007, 154, E31-E35.

11. The ratio of $\mathbf{1 2}$ cis and $\mathbf{1 2}$ trans was determined on the basis of the NMR spectrum of $\mathbf{1 2} \mathbf{b}_{\text {trans }}$; Williams, S. J.; Hoos, R.; Withers, S. G. J. Am. Chem. Soc. 2000, 122, 2223-2235.

12. (a) Shono, T.; Matsumura, Y.; Inoue, K. J. Chem. Soc., Chem. Commun. 1983, 1169-1171; (b) Matsumura, Y.; Nakamura, Y.; Maki, T.; Onomura, O. Tetrahedron Lett. 2000, 41, 7685-7689.

13. Methoxylated compound $\mathbf{1 4}$ purified with silica gel column chromatography was transformed into a certain amount of unsaturated compound $\mathbf{1 5}$ as a by-product. Accordingly the yield of $\mathbf{1 5}$ by two steps without purification of $\mathbf{1 4}$ was better than that with purification of $\mathbf{1 4}$. The yield of $\mathbf{1 7}$ was improved without purification of the corresponding methoxylated compound.

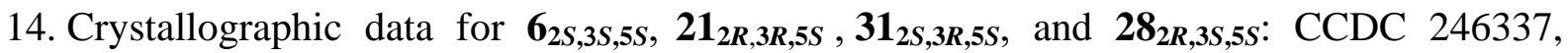
246338, 746282, and 746283, contain the supplementary crystallographic data for this paper. The data can be obtained free of charge via www.ccdc.cam.ac.uk/data_request/cif, by emailing data_request@ccdc.cam.ac.uk, or by contacting The Cambridge Crystallographic Data Centre, 12, Union Road, Cambridge CB2 1EZ, UK; fax: +44(0)-1223-336033.

15. (a) Matsumura, Y.; Tomita, T. Tetrahedron Lett. 1994, 35, 3737-3740; (b) Matsumura, Y.; Yoshimoto, Y.; Horikawa, C. Maki, T.; Watanabe, M. Tetrahedron Lett. 1996, 37, 5715-5718; (c) Matsumura, Y.; Asano, T.; Nakagiri, T.; Onomura, O. J. Chin. Chem. Soc. 1998, 45, 297-302.

16. The allylic 1,3-strain in $\mathbf{F}$ may compel acetoxymethyl group at the 5-position quasiaxial: (a) Hoffmann, R. W. Chem. Rev. 1989, 89, 1841-1860; (b) Momose, T.; Toyooka, N. J. Org. Chem. 1994, 59, 943-945; (c) Matsumura, Y.; Inoue, M.; Nakamura, Y.; Talib, I. L., Maki, T.; Onomura, O. Tetrahedron Lett. 2000, 41, 4619-4622. 